\title{
Bogoliubov Theory of Dipolar Bose Gas in Weak Random Potential
}

\author{
Mahmoud Ghabour ${ }^{1}$ and Axel Pelster ${ }^{2,3}$ \\ ${ }^{1}$ Insitute für Theoretische Physik, Freie Universität Berlin, Arnimallee 14, 14195 Berlin, Germany \\ ${ }^{2}$ Physics Department and Research Center OPTIMAS, \\ Technical University of Kaiserslautern, 67663 Kaiserslautern, Germany \\ ${ }^{3}$ Hanse-Wissenschaftskolleg, Lehmkuhlenbusch 4, D-27733 Delmenhorst, Germany
}

\begin{abstract}
We consider a dilute homogeneous Bose gas with both an isotropic short-range contact interaction and an anisotropic long-range dipole-dipole interaction in a weak random potential at low temperature in three dimensions. Within the realm of Bogoliubov theory we analyze how both condensate and superfluid are depleted due to quantum and thermal fluctuations as well as disorder fluctuations.
\end{abstract}

PACS numbers: 03.75.Hh, 67.85.De

\section{INTRODUCTION}

Since the first observation of Bose-Einstein condensation (BEC) in 1995 for alkali atomic vapors of ${ }^{87} \mathrm{Rb}$ and ${ }^{23} \mathrm{Na}$ atoms both theoretical and experimental research has accelerated to study this newly discovered macroscopic quantum phenomenon, where a fraction of these bosons occupies the same quantum mechanical ground state. Achieving BEC in experiment for alkali atoms was only possible due to the discovery of efficient cooling and trapping techniques which became available by the end of the twentieth century [1-3]. With more cooling advances, research interest has not only increased in the field of ultra-cold quantum gases with isotropic short-range contact interaction, which represents the effective interaction between atoms and is dominated by the s-wave scattering length. Also the anisotropic long-range dipole-dipole interaction has been made accessible to detailed study by the formation of a BEC in a dipolar quantum gas of ${ }^{52} \mathrm{Cr}$ atoms [4, 5]. Further atomic BECs with magnetic dipole-dipole interaction followed soon with ${ }^{164}$ Dy [6] and ${ }^{168} \operatorname{Er}$ [7] atoms. Strong electric dipole-dipole interactions were realized by a stimulated Raman adiabatic passage (STIRAP) experiment, which allowed the creation of ${ }^{40} \mathrm{~K}$ ${ }^{87} \mathrm{Rb}$ molecules in the rovibrational ground state [8, 9]

The research field of ultra-cold quantum gases allows today to analyze many condensed matter problems in the spirit of Feynman's quantum simulator [10], as all ingredients of the quantum many-body Hamiltonian can be experimentally tuned with an unprecedented precision. The kinetic energy can be controlled with spin-orbit coupling [11, 12], the potential energy can be harmonic [13] box-like [14] or even anharmonic [15] and also the strength of contact as well as dipolar interaction are tunable [16 18]. In addition, in order to make quantum gas simulators even more realistic, various experimental and theoretical methods for controlling the effect of disorder have been designed. Using superfluid helium in vycor glass, which represents some kind of porous media, the random distribution of pores represents a random environment [19]. Laser speckles are produced by focusing the laser beam on a glass plate, where the resulting random interference pattern is reflected to a BEC cell [20-
23]. Wire traps represent magnetic traps on atomic chips where the roughness and the imperfection of the wire surface generates a disorder potential [24, 25]. Another possibility to create a random potential is to trap one species of atoms randomly in a deep optical lattice, which serves as frozen scatterers for a second atomic species [26, 27]. In addition also, incommensurable lattices provide a useful random environment [28 30].

In order to study the properties of interacting bosons in such a random potential Huang and Meng proposed a Bogoliubov theory, which was applied to the case of superfluid helium in porous media [31], and extended later by others $[32-35]$. For a delta-correlated disorder it was found that both a condensate and a superfluid depletion occurs due to the localization of bosons in the respective minima of the external random potential which is present even at zero temperature. A generalization to the corresponding situation, where the disorder correlation function falls off with a characteristic correlation length as, for instance, a Gauss function [36, 37], laser speckles [38] or a Lorentzian [39] is straightforward.

In this paper we extend the Bogoliubov theory of Huang and Meng [31] to a dipolar Bose gas at finite temperature. With this we go beyond the zero-temperature mean-field approach where the Gross-Pitaevskii equation is solved perturbativly with respect to the random potential [37, 39]. This extension to the finite-temperature regime allows us to study in detail how the anisotropy of superfluidity can be tuned, a phenomenon which also occurs at zero-temperature [37, 39], but turns out to be enhanced by the thermal fluctuations. We observe in addition that contact and dipolar interaction have different effects upon quantum, thermal, and disorder depletion of condensate and superfluid.

The paper is organized as follows. In Section. II we revisit the Bogoliubov theory of the homogeneous dirty boson problem. With this, we determine for a general two-particle interaction and a general disorder correlation for different observables the beyond mean-field corrections which stem from quantum, thermal, and disorder fluctuations. In Section. III we specialize our treatment for a dipolar Bose gas and a delta-correlated disorder in the zero-temperature and the thermodynamic limit. 
In particular, we investigate the particle and condensate density as well as the inner energy. In Section. IV we extend the Bogoliubov theory in order to derive the superfluid depletion which turns out to be only due to the external random potential and the thermal excitation. In Section. $\mathrm{V}$ we consider how finite-temperature effects on the condensate depletion as well as the normal fluid component depend on the respective strength of contact and dipolar interaction.

\section{BOGOLIUBOV THEORY}

A three-dimensional ultra-cold dipolar Bose gas in a weak random potential is modeled by the grand-canonical Hamiltonian

$$
\begin{aligned}
\hat{\mathcal{K}}= & \int d^{3} \mathbf{x} \hat{\psi}^{\dagger}(\mathbf{x})\left[\frac{-\hbar^{2} \nabla^{2}}{2 m}-\mu+U(\mathbf{x})\right] \hat{\psi}(\mathbf{x}) \\
& +\frac{1}{2} \int d^{3} \mathbf{x} \int d^{3} \mathbf{x}^{\prime} \hat{\psi}^{\dagger}(\mathbf{x}) \hat{\psi}^{\dagger}\left(\mathbf{x}^{\prime}\right) V\left(\mathbf{x}, \mathbf{x}^{\prime}\right) \hat{\psi}\left(\mathbf{x}^{\prime}\right) \hat{\psi}(\mathbf{x})
\end{aligned}
$$

where $\hat{\psi}(\mathbf{x})$ and $\hat{\psi}^{\dagger}(\mathbf{x})$ are the usual field operators for Bose particles of mass $m$, which satisfy the following commutation relations

$$
\begin{aligned}
{\left[\hat{\psi}(\mathbf{x}), \hat{\psi}^{\dagger}\left(\mathbf{x}^{\prime}\right)\right] } & =\delta\left(\mathbf{x}-\mathbf{x}^{\prime}\right) \\
{\left[\hat{\psi}(\mathbf{x}), \hat{\psi}\left(\mathbf{x}^{\prime}\right)\right] } & =\left[\hat{\psi}^{\dagger}(\mathbf{x}), \hat{\psi}^{\dagger}\left(\mathbf{x}^{\prime}\right)\right]=0 .
\end{aligned}
$$

Here $\mu$ denotes the chemical potential and $U(\mathbf{x})$ represents the random potential. Irrespective of the physical origin of the disorder potential, we assume that the average over the random potential vanishes and that it has some kind of correlation i.e. $\langle U(\mathbf{x})\rangle=0$ and $\left\langle U(\mathbf{x}) U\left(\mathbf{x}^{\prime}\right)\right\rangle=R\left(\mathbf{x}-\mathbf{x}^{\prime}\right)$. Furthermore, the two-particle interaction potential for a dipolar Bose gas consists of two different parts: $V\left(\mathbf{x}, \mathbf{x}^{\prime}\right)=V_{\delta}\left(\mathbf{x}-\mathbf{x}^{\prime}\right)+V_{\mathrm{dd}}\left(\mathbf{x}-\mathbf{x}^{\prime}\right)$. On the one hand the short-range isotropic contact interaction is given by $V_{\delta}(\mathbf{x})=g \delta(\mathbf{x})$, where $g=4 \pi a \hbar^{2} / m$ represents its strength with the s-wave scattering length $a$. From now on we assume a positive $a$, i.e. a repulsive contact interaction, which depletes the particles from the ground state due to quantum and thermal fluctuations and also forbids them from being localized in the minima of the external random potential $U(\mathbf{x})$. Thus, unlike the case of free fermions, where Pauli blocking prevents the particles from localizing in a single orbital, bosons require a repulsive interaction to prevent them from collapsing into the respective minima of the external random potential. On the other hand the long-range anisotropic polarized dipolar part is written in real space for dipoles aligned along $z$-axis direction according to

$$
V_{\mathrm{dd}}(\mathbf{x})=\frac{C_{\mathrm{dd}}}{4 \pi} \frac{\left(x^{2}+y^{2}+z^{2}\right)-3 z^{2}}{\left(x^{2}+y^{2}+z^{2}\right)^{5 / 2}},
$$

where $C_{\mathrm{dd}}$ represents the dipolar interaction strength due to magnetic or electric dipole moments. In the first case we have $C_{\mathrm{dd}}=\mu_{0} d_{\mathrm{m}}^{2}$, with the magnetic dipole moment $d_{\mathrm{m}}$ and the magnetic permeability of vacuum $\mu_{0}$, and in the latter case we have $C_{\mathrm{dd}}=d_{\mathrm{e}}^{2} / \epsilon_{0}$, with the electric dipole moment $d_{\mathrm{e}}$ and the vacuum dielectric constant $\epsilon_{0}$. Note that, due to the anisotropic character of the dipolar interaction, many static and dynamic properties of a dipolar Bose gas become tunable [4, 5, 40, 41], consequences for a random environment have only recently be explored [37, 39].

With the help of a Fourier transformation we can rewrite the Hamiltonian (1) in momentum space according to

$$
\begin{aligned}
\hat{\mathcal{K}}= & \sum_{\mathbf{k}}\left(\frac{\hbar^{2} \mathbf{k}^{2}}{2 m}-\mu\right) \hat{a}_{\mathbf{k}}^{\dagger} \hat{a}_{\mathbf{k}}+\frac{1}{v} \sum_{\mathbf{p}, \mathbf{k}} U_{\mathbf{p}-\mathbf{k}} \hat{a}_{\mathbf{p}}^{\dagger} \hat{a}_{\mathbf{k}} \\
& +\frac{1}{2 v} \sum_{\mathbf{p}, \mathbf{k}, \mathbf{q}} V_{\mathbf{q}} \hat{a}_{\mathbf{k}+\mathbf{q}}^{\dagger} \hat{a}_{\mathbf{p}-\mathbf{q}}^{\dagger} \hat{a}_{\mathbf{p}} \hat{a}_{\mathbf{k}},
\end{aligned}
$$

where $v$ denotes the volume and the interaction potential in momentum space is given by [17]

$$
V_{\mathbf{q}}=g+\frac{C_{\mathrm{dd}}}{3}\left(3 \cos ^{2} \theta-1\right)
$$

Here $\theta$ denotes the angle between the polarization direction, which is here along the $z$-axis, and the wave vector q. Note that (5) is not continuous at $\mathbf{q}=\mathbf{0}$, as the limit $\mathbf{q} \rightarrow \mathbf{0}$ is direction dependent. This is the origin for various anisotropic properties, which are characteristic for dipolar Bose gases [37, 39, 42, 43]. The operators $\hat{a}_{\mathbf{k}}$ and $\hat{a}_{\mathbf{k}}^{\dagger}$ are the annihilation and creation operators in Fourier space, respectively, which turn out to satisfy the bosonic commutation relations

$$
\left[\hat{a}_{\mathbf{k}}, \hat{a}_{\mathbf{k}^{\prime}}^{\dagger}\right]=\delta_{\mathbf{k k}^{\prime}},\left[\hat{a}_{\mathbf{k}}, \hat{a}_{\mathbf{k}^{\prime}}\right]=\left[\hat{a}_{\mathbf{k}}^{\dagger}, \hat{a}_{\mathbf{k}^{\prime}}^{\dagger}\right]=0
$$

Near absolute zero temperature the number of the particles $N_{\mathbf{0}}$ in the ground state $\left|\Phi_{\mathbf{0}}\right\rangle$ becomes macroscopically large. In this case we have due to (6) $\hat{a}_{\mathbf{0}}\left|\Phi_{\mathbf{0}}\right\rangle \approx \sqrt{N_{0}}\left|\Phi_{\mathbf{0}}\right\rangle$ and $\hat{a}_{\mathbf{0}}^{\dagger}\left|\Phi_{\mathbf{0}}\right\rangle \approx \sqrt{N_{\mathbf{0}}}\left|\Phi_{\mathbf{0}}\right\rangle$, so the operators $\hat{a}_{\mathbf{0}}$ and $\hat{a}_{\mathbf{0}}^{\dagger}$ approximately commute with each other. Thus we can apply the Bogoliubov prescription [44] and replace the creation and annihilation operators of the ground state $\mathbf{k}=\mathbf{0}$ by a c-number, i.e. $\hat{a}_{\mathbf{0}}=\hat{a}_{\mathbf{0}}^{\dagger}=\sqrt{N_{\mathbf{0}}}$. As a consequence, we have to decompose the respective momentum summations in the Hamiltonian (44) into their ground state $\mathbf{k}=\mathbf{0}$ and excited states $\mathbf{k} \neq \mathbf{0}$ contributions. By doing so, we perform the following two physical approximations. At first, we ignore terms which contain creation and annihilation operators of the excited states $\mathbf{k} \neq \mathbf{0}$, which are of third and fourth order, as they represent higher-order interactions of the particles out of the condensate. Such an approximation is justified in the case of weakly interacting systems. Secondly, we assume for weak enough disorder that disorder fluctuations decouple in lowest order. Therefore, we ignore the terms 
$U_{\mathbf{p}-\mathbf{k}} \hat{a}_{\mathbf{p}}^{\dagger} \hat{a}_{\mathbf{k}}$ with both $\mathbf{k} \neq \mathbf{0}$ and $\mathbf{p} \neq \mathbf{0}$. Note that these two physical approximations imply that the excited states are only rarely occupied, i.e. $N-N_{\mathbf{0}} \ll N$. With this we get the simplified Hamiltonian

$$
\begin{aligned}
\hat{\mathcal{K}}^{\prime}= & \left(-\mu+\frac{1}{v} U_{0}\right) N_{\mathbf{0}}+\frac{1}{2 v} V_{\mathbf{0}} N_{\mathbf{0}}^{2} \\
& +\frac{1}{2} \sum_{\mathbf{k}}^{\prime}\left(\frac{\hbar^{2} \mathbf{k}^{2}}{2 m}-\mu\right)\left(\hat{a}_{\mathbf{k}}^{\dagger} \hat{a}_{\mathbf{k}}+\hat{a}_{-\mathbf{k}}^{\dagger} \hat{a}_{-\mathbf{k}}\right) \\
& +\frac{1}{v} \sqrt{N_{\mathbf{0}}} \sum_{\mathbf{k}}^{\prime} U_{\mathbf{k}, \mathbf{0}}\left(\hat{a}_{\mathbf{k}}^{\dagger}+\hat{a}_{-\mathbf{k}}\right) \\
& +\frac{1}{2 v} N_{\mathbf{0}} \sum_{\mathbf{k}}^{\prime}\left(V_{\mathbf{0}}+V_{\mathbf{k}}\right)\left(\hat{a}_{\mathbf{k}}^{\dagger} \hat{a}_{\mathbf{k}}+\hat{a}_{-\mathbf{k}}^{\dagger} \hat{a}_{-\mathbf{k}}\right) \\
& +\frac{1}{2 v} N_{\mathbf{0}} \sum_{\mathbf{k}}^{\prime} V_{\mathbf{k}}\left(\hat{a}_{\mathbf{k}}^{\dagger} \hat{a}_{-\mathbf{k}}^{\dagger}+\hat{a}_{\mathbf{k}} \hat{a}_{-\mathbf{k}}\right) .
\end{aligned}
$$

Here the prime over the summation symbol indicates that the ground state $\mathbf{k}=\mathbf{0}$ is excluded. The appearance of off-diagonal terms $\hat{a}_{\mathbf{k}} \hat{a}_{-\mathbf{k}}$ and $\hat{a}_{\mathbf{k}}^{\dagger} \hat{a}_{-\mathbf{k}}^{\dagger}$ with $\mathbf{k} \neq \mathbf{0}$ in equation (7) indicates that the state $\mathbf{k}=\mathbf{0}$ is no longer the ground state of the interacting system. In order to determine this new mean-field ground state we have to diagonalize the Hamiltonian (77). To this end we follow Ref. [31] and use the inhomogeneous Bogoliubov transformation

$$
\begin{aligned}
& \hat{a}_{\mathbf{k}}=u_{\mathbf{k}} \hat{\alpha}_{\mathbf{k}}-v_{\mathbf{k}} \hat{\alpha}_{-\mathbf{k}}^{\dagger}-z_{\mathbf{k}}, \\
& \hat{a}_{\mathbf{k}}^{\dagger}=u_{\mathbf{k}} \hat{\alpha}_{\mathbf{k}}^{\dagger}-v_{\mathbf{k}} \hat{\alpha}_{-\mathbf{k}}-z_{\mathbf{k}}^{*} .
\end{aligned}
$$

Note that the Bogoliubov amplitudes $u_{\mathbf{k}}$ and $v_{\mathbf{k}}$ can be chosen to be real without loss of generality. Furthermore, we impose upon the new operators $\hat{\alpha}_{\mathbf{k}}, \hat{\alpha}_{\mathbf{k}}^{\dagger}$ that they also satisfy bosonic commutation relations

$$
\left[\hat{\alpha}_{\mathbf{k}}, \hat{\alpha}_{\mathbf{k}^{\prime}}^{\dagger}\right]=\delta_{\mathbf{k k}^{\prime}},\left[\hat{\alpha}_{\mathbf{k}}, \hat{\alpha}_{\mathbf{k}^{\prime}}\right]=\left[\hat{\alpha}_{\mathbf{k}}^{\dagger}, \hat{\alpha}_{\mathbf{k}^{\prime}}^{\dagger}\right]=0 .
$$

Inserting (8) in the simplified Hamiltonian (7) we obtain via diagonalization the following results for the Bogoliubov parameters

$$
\begin{aligned}
& u_{\mathbf{k}}^{2}=\frac{1}{2}\left[\frac{\frac{\hbar^{2} \mathbf{k}^{2}}{2 m}-\mu+n_{\mathbf{0}}\left(V_{\mathbf{0}}+V_{\mathbf{k}}\right)}{E_{\mathbf{k}}}+1\right], \\
& v_{\mathbf{k}}^{2}=\frac{1}{2}\left[\frac{\frac{\hbar^{2} \mathbf{k}^{2}}{2 m}-\mu+n_{\mathbf{0}}\left(V_{\mathbf{0}}+V_{\mathbf{k}}\right)}{E_{\mathbf{k}}}-1\right],
\end{aligned}
$$

and the translations

$$
z_{\mathbf{k}}=\frac{\frac{1}{v} \sqrt{N_{\mathbf{0}}} U_{\mathbf{k}}\left(\frac{\hbar^{2} \mathbf{k}^{2}}{2 m}-\mu+n_{\mathbf{0}} V_{\mathbf{0}}\right)}{E_{\mathbf{k}}^{2}} .
$$

Here we have introduced for brevity the condensate density $n_{\mathbf{0}}=N_{\mathbf{0}} / v$ and the quasi-particle dispersion

$$
E_{\mathbf{k}}=\sqrt{\left[\frac{\hbar^{2} \mathbf{k}^{2}}{2 m}-\mu+n_{\mathbf{0}}\left(V_{\mathbf{0}}+V_{\mathbf{k}}\right)\right]^{2}-\left(n_{\mathbf{0}} V_{\mathbf{k}}\right)^{2}} .
$$

Note that the diagonalized Hamiltonian changes with each realization of the disorder potential $U(\mathbf{x})$. Therefore, we get the final Hamiltonian of the dirty dipolar Bose system by performing the disorder ensemble average

$$
\begin{aligned}
\left\langle\hat{\mathcal{K}}^{\prime}\right\rangle= & v\left(-\mu n_{\mathbf{0}}+\frac{1}{2} V_{\mathbf{0}} n_{\mathbf{0}}^{2}\right) \\
& +\frac{1}{2} \sum_{\mathbf{k}}^{\prime}\left\{E_{\mathbf{k}}-\left[\frac{\hbar^{2} \mathbf{k}^{2}}{2 m}-\mu+n_{\mathbf{0}}\left(V_{\mathbf{0}}+V_{\mathbf{k}}\right)\right]\right\} \\
& +\frac{1}{2} \sum_{\mathbf{k}}^{\prime} E_{\mathbf{k}}\left(\hat{\alpha}_{\mathbf{k}}^{\dagger} \hat{\alpha}_{\mathbf{k}}+\hat{\alpha}_{-\mathbf{k}}^{\dagger} \hat{\alpha}_{-\mathbf{k}}\right) \\
& -\sum_{\mathbf{k}}^{\prime} \frac{n_{\mathbf{0}} R_{\mathbf{k}}}{E_{\mathbf{k}}^{2}}\left(\frac{\hbar^{2} \mathbf{k}^{2}}{2 m}-\mu+n_{\mathbf{0}} V_{\mathbf{0}}\right)
\end{aligned}
$$

With this it is straightforward to determine the corresponding grand-canonical potential $\Omega_{\mathrm{eff}}=-\beta^{-1} \ln \mathcal{Z}_{G}$, where $\mathcal{Z}_{G}=\operatorname{Tr} e^{-\beta\left\langle\hat{\mathcal{K}}^{\prime}\right\rangle}$ denotes the grand-canonical partition function and $\beta=1 /\left(k_{\mathrm{B}} T\right)$ is the reciprocal temperature:

$$
\begin{aligned}
\Omega_{\mathrm{eff}}=v & \left(-\mu n_{\mathbf{0}}+\frac{1}{2} V_{\mathbf{0}} n_{\mathbf{0}}^{2}\right) \\
& +\frac{1}{2} \sum_{\mathbf{k}}^{\prime}\left\{E_{\mathbf{k}}-\left[\frac{\hbar^{2} \mathbf{k}^{2}}{2 m}-\mu+n_{\mathbf{0}}\left(V_{\mathbf{0}}+V_{\mathbf{k}}\right)\right]\right\} \\
& +\sum_{\mathbf{k}}^{\prime} \frac{1}{\beta} \ln \left(1-e^{-\beta E_{\mathbf{k}}}\right) \\
& -\sum_{\mathbf{k}}^{\prime} \frac{n_{\mathbf{0}} R_{\mathbf{k}}}{E_{\mathbf{k}}^{2}}\left(\frac{\hbar^{2} \mathbf{k}^{2}}{2 m}-\mu+n_{\mathbf{0}} V_{\mathbf{0}}\right) .
\end{aligned}
$$

Extremizing equation (14) with respect to the condensate density $n_{\mathbf{0}}$ for fixed chemical potential $\mu$ we find up to first order in quantum, thermal, and disorder fluctuations

$$
\begin{aligned}
n_{\mathbf{0}}=\frac{\mu}{V_{\mathbf{0}}}- & \frac{1}{2 v} \sum_{\mathbf{k}}^{\prime}\left\{\frac{\left(\frac{\hbar^{2} \mathbf{k}^{2}}{2 m}-\mu\right)\left(V_{\mathbf{0}}+V_{\mathbf{k}}\right)}{V_{\mathbf{0}} E_{\mathbf{k}}}\right. \\
& \left.\frac{+\frac{\mu}{V_{\mathbf{0}}}\left(V_{\mathbf{0}}^{2}+2 V_{\mathbf{0}} V_{\mathbf{k}}\right)-E_{\mathbf{k}}\left(V_{\mathbf{0}}+V_{\mathbf{k}}\right)}{V_{\mathbf{0}} E_{\mathbf{k}}}\right\} \\
& -\frac{1}{v} \sum_{\mathbf{k}}^{\prime} \frac{\left(\frac{\hbar^{2} \mathbf{k}^{2}}{2 m}-\mu\right)\left(V_{\mathbf{0}}+V_{\mathbf{k}}\right)+\frac{\mu}{V_{\mathbf{0}}}\left(V_{\mathbf{0}}^{2}+2 V_{\mathbf{0}} V_{\mathbf{k}}\right)}{\left(e^{\beta E_{\mathbf{k}}}-1\right) V_{\mathbf{0}} E_{\mathbf{k}}} \\
& +\frac{1}{v} \sum_{\mathbf{k}}^{\prime} \frac{R_{\mathbf{k}}}{E_{\mathbf{k}}^{4}}\left\{\frac{1}{V_{\mathbf{0}}}\left(\frac{\hbar^{2} \mathbf{k}^{2}}{2 m}\right)^{3}-\frac{\mu}{V_{\mathbf{0}}}\left(\frac{\hbar^{2} \mathbf{k}^{2}}{2 m}\right)^{2}\right\},
\end{aligned}
$$


where the quasi-particle dispersion (12) now reads

$$
E_{\mathbf{k}}=\sqrt{\left(\frac{\hbar^{2} \mathbf{k}^{2}}{2 m}\right)^{2}+\mu \frac{\hbar^{2} \mathbf{k}^{2}}{m} \frac{V_{\mathbf{k}}}{V_{\mathbf{0}}}} .
$$

Note that the zeroth order $n_{\mathbf{0}}=\mu / V_{\mathbf{0}}$ in (15) represents the mean-field result and corresponds to the HugenholtzPines relation [45]. It makes the quasi-particle dispersion (12) according to (16) linear and gapless (massless) in the long-wavelength limit $\mathbf{k} \rightarrow \mathbf{0}$, in accordance with the Nambu-Goldstone theorem [46, 47].

Inserting (15) in (14) the grand-canonical potential $\Omega_{\text {eff }}$ reduces up to first order in all fluctuations to the grand-canonical free energy

$$
\begin{aligned}
\mathcal{F}= & -\frac{v \mu^{2}}{2 V_{\mathbf{0}}} \\
& +\frac{1}{2} \sum_{\mathbf{k}}^{\prime}\left[E_{\mathbf{k}}-\left(\frac{\hbar^{2} \mathbf{k}^{2}}{2 m}+\mu \frac{V_{\mathbf{k}}}{V_{\mathbf{0}}}\right)\right] \\
& +\sum_{\mathbf{k}}^{\prime} \frac{1}{\beta} \ln \left(1-e^{-\beta E_{\mathbf{k}}}\right) \\
& -\sum_{\mathbf{k}}^{\prime} \frac{R_{\mathbf{k}}}{E_{\mathbf{k}}^{2}} \frac{\hbar^{2} \mathbf{k}^{2}}{2 m} \frac{\mu}{V_{\mathbf{0}}} .
\end{aligned}
$$

The particle number density follows then from equation (17) via the thermodynamic relation $n=-\frac{1}{v} \frac{\partial \mathcal{F}}{\partial \mu}$ :

$$
\begin{aligned}
n= & \frac{\mu}{V_{\mathbf{0}}}-\frac{1}{2 v} \sum_{\mathbf{k}}^{\prime}\left(\frac{\frac{\hbar^{2} \mathbf{k}^{2}}{2 m} \frac{V_{\mathbf{k}}}{V_{\mathbf{0}}}}{E_{\mathbf{k}}}-\frac{V_{\mathbf{k}}}{V_{\mathbf{0}}}\right) \\
& -\frac{1}{v} \sum_{\mathbf{k}}^{\prime} \frac{\frac{\hbar^{2} \mathbf{k}^{2}}{2 m} \frac{V_{\mathbf{k}}}{V_{\mathbf{0}}}}{\left(e^{\beta E_{\mathbf{k}}}-1\right) E_{\mathbf{k}}} \\
& +\frac{1}{v} \sum_{\mathbf{k}}^{\prime} \frac{R_{\mathbf{k}}}{E_{\mathbf{k}}^{4}} \frac{1}{V_{\mathbf{0}}}\left(\frac{\hbar^{2} \mathbf{k}^{2}}{2 m}\right)^{3} .
\end{aligned}
$$

Eliminating from (15) and (18) the chemical potential $\mu$ allows to determine the condensate depletion up to first order in the respective fluctuations:

$$
n-n_{\mathbf{0}}=n^{\prime}+n_{\mathrm{th}}+n_{\mathrm{R}} .
$$

Thus the total particle density consists of four parts. Within the Bogoliubov theory the main contribution is due to the condensate density $n_{\mathbf{0}}$, whereas the occupation of the excited states above the ground state consists of three distinct terms: the condensate depletion due to interaction and quantum fluctuations is denoted by [48]

$$
n^{\prime}=\frac{1}{2 v} \sum_{\mathbf{k}}^{\prime}\left(\frac{\frac{\hbar^{2} \mathbf{k}^{2}}{2 m}+n V_{\mathbf{k}}}{E_{\mathbf{k}}}-1\right) .
$$

Note that we neglected here any impact of the external random potential upon the quantum fluctuations, an interesting case which was only recently studied in detail in Refs. [49, 50].
The condensate depletion due to the thermal fluctuations is given by

$$
n_{\mathrm{th}}=\frac{1}{v} \sum_{\mathbf{k}}^{\prime} \frac{\frac{\hbar^{2} \mathbf{k}^{2}}{2 m}+n V_{\mathbf{k}}}{E_{\mathbf{k}}} \frac{1}{e^{\beta E_{\mathbf{k}}}-1} .
$$

And finally, the condensate depletion due to the external random potential results in

$$
n_{\mathrm{R}}=\frac{1}{v} \sum_{\mathbf{k}}^{\prime} \frac{n R_{\mathbf{k}}}{E_{\mathbf{k}}^{4}}\left(\frac{\hbar^{2} \mathbf{k}^{2}}{2 m}\right)^{2} .
$$

Note that the validity of our approximation depends on the condition $n-n_{\mathbf{0}} \ll n$, i.e. the depletion is small, so that condensate density $n_{\mathbf{0}}$ and total density $n$ are approximately the same. With this dispersion (16) in all fluctuation terms (20)-(22) reduces to

$$
E_{\mathbf{k}}=\sqrt{\left(\frac{\hbar^{2} \mathbf{k}^{2}}{2 m}\right)^{2}+n V_{\mathbf{k}} \frac{\hbar^{2} \mathbf{k}^{2}}{m}}
$$

which represents the celebrated Bogoliubov spectrum for the collective excitations. Note that in the Popov approximation the particle density $n$ in (23) is substituted by the condensate density $n_{\mathbf{0}}$, so that this Bogoliubov dispersion acquires fluctuation corrections [51].

The grand-canonical ground-state energy $E-\mu N$ follows directly from equation (17), by using the thermodynamic relation $E-\mu N=-T^{2}\left(\frac{\partial}{\partial T} \frac{\mathcal{F}}{T}\right)_{v, \mu}$ :

$$
E-\mu N=-\frac{v \mu^{2}}{2 V_{0}}+E^{\prime}+E_{\mathrm{th}}+E_{\mathrm{R}}
$$

The first term on the right-hand side of (24) represents the ground-state mean-field energy for the homogeneous Bose-Einstein condensate, whereas the other three terms denote energy contributions which are due to the partial occupation of the excited states. The energy shift due to interaction and quantum fluctuations is denoted by

$$
E^{\prime}=\frac{1}{2} \sum_{\mathbf{k}}^{\prime}\left[E_{\mathbf{k}}-\left(\frac{\hbar^{2} \mathbf{k}^{2}}{2 m}+n V_{\mathbf{k}}\right)\right] .
$$

The energy shift due to the thermal fluctuations is given by

$$
E_{\mathrm{th}}=\sum_{\mathbf{k}}^{\prime} E_{\mathbf{k}} \frac{1}{e^{\beta E_{\mathbf{k}}-1}} .
$$

And, finally, the energy shift due to the external random potential reads

$$
E_{\mathrm{R}}=-\sum_{\mathbf{k}}^{\prime} \frac{R_{\mathbf{k}}}{E_{\mathbf{k}}^{2}} \frac{\hbar^{2} \mathbf{k}^{2}}{2 m} n .
$$

Within the next section these general results are further evaluated and discussed for a dipolar Bose gas. 


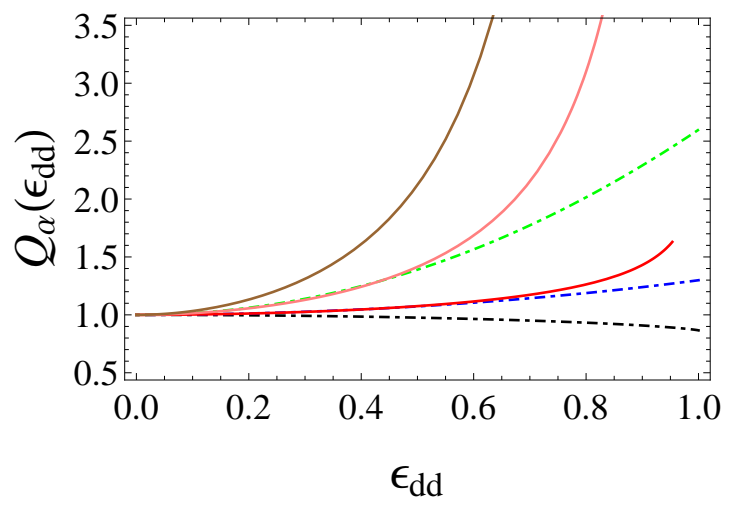

Figure 1: (color online). Dipolar enhancement function $Q_{\alpha}\left(\epsilon_{\mathrm{dd}}\right)$ versus relative dipolar interaction strength $\epsilon_{\mathrm{dd}}$ for different values of $\alpha$ : $-5 / 2$ (brown, upper solid), $-3 / 2$ (pink, middle solid), $-1 / 2$ (red, lower solid), $1 / 2$ (black, lower dotteddashed), $3 / 2$ (blue, middle dotted-dashed), 5/2 (green, upper dotted-dashed).

\section{ZERO-TEMPERATURE RESULTS}

In this section we specialize our results to the zerotemperature limit for a delta-correlated random potential $R\left(\mathbf{x}-\mathbf{x}^{\prime}\right)=R_{0} \delta\left(\mathbf{x}-\mathbf{x}^{\prime}\right)$, where $R_{0}$ denotes the disorder strength. In the thermodynamic limit, when both the particle number $N$ and the volume $v$ diverge, i.e. $N \rightarrow \infty$ and $v \rightarrow \infty$, but their ratio $n=\frac{N}{v}$ remains constant, the respective wave vector sums converge towards integrals: $\frac{1}{v} \sum_{\mathbf{k}}^{\prime} \rightarrow \int \frac{d^{3} \mathbf{k}}{(2 \pi)^{3}}$. With this we obtain for the quantum depletion (20) the concrete result [42, 43]

$$
n^{\prime}=n_{\mathrm{B}} Q_{\frac{3}{2}}\left(\epsilon_{\mathrm{dd}}\right),
$$

with the original Bogoliubov expression [4]

$$
n_{\mathrm{B}}=\frac{8}{3 \sqrt{\pi}}(n a)^{\frac{3}{2}}
$$

whereas the disorder depletion (22) specializes to [37, 39]

$$
n_{\mathrm{R}}=n_{\mathrm{HM}} Q_{-\frac{1}{2}}\left(\epsilon_{\mathrm{dd}}\right) .
$$

Here $\epsilon_{\mathrm{dd}}=C_{\mathrm{dd}} / 3 g$ denotes the relative dipolar interaction strength, and

$$
n_{\mathrm{HM}}=\frac{m^{2} R_{0}}{8 \hbar^{4} \pi^{\frac{3}{2}}} \sqrt{\frac{n}{a}},
$$

represents the Huang-Meng result for the isotropic contact interaction [31]. In addition, the functions

$$
Q_{\alpha}\left(\epsilon_{\mathrm{dd}}\right)=\int_{0}^{1}\left[1+\epsilon_{\mathrm{dd}}\left(3 x^{2}-1\right)\right]^{\alpha} d x,
$$

which describe the dipolar effect, can be expressed analytically [52]

$$
Q_{\alpha}\left(\epsilon_{\mathrm{dd}}\right)=\left(1-\epsilon_{\mathrm{dd}}\right)^{\alpha}{ }_{2} F_{1}\left(-\alpha, \frac{1}{2} ; \frac{3}{2} ; \frac{-3 \epsilon_{\mathrm{dd}}}{1-\epsilon_{\mathrm{dd}}}\right)
$$

in terms of the hypergeometric function ${ }_{2} F_{1}$. The dipolar function $Q_{\alpha}\left(\epsilon_{\mathrm{dd}}\right)$ is depicted in Fig. 1, and increases for increasing magnitude of $\alpha$ for both positive and negative sign. Note that, in order to avoid any instability, we restricted $\epsilon_{\mathrm{dd}}$ in Fig. 1 to the maximum value one, so that the radicand in the Bogoliubov spectrum (23) remains positive when $\mathbf{k} \rightarrow 0$ [42, 43].

We conclude that Eqs. (28), (30) reproduce the contact interaction results Eqs. (29), (31) for $\epsilon_{\mathrm{dd}}=0$ due to the property $Q_{\alpha}(0)=1$. Note that increasing the repulsive contact interaction has opposite effects on the zero-temperature condensate depletion. On the one hand more and more bosons are then scattered from the ground state to some excited states so that the quantum depletion (29) increases. On the other hand the localization of bosons in the respective minima of the random potential is hampered, so that disorder depletion (31) decreases. In contrast to that the long-range dipolar interaction turns out to enhance both the quantum deletion (28) and the disorder depletion (30), see Fig. 11 As the dipolar interaction potential (3) has two repulsive and only one attractive direction in real space, it yields a net expulsion of bosons from the ground state which is described by $Q_{\frac{3}{2}}\left(\epsilon_{\mathrm{dd}}\right)$ in (28). In contrast to that the dipolar interaction supports the localization of bosons in the random environment [37, 39] according to $Q_{-\frac{1}{2}}\left(\epsilon_{\mathrm{dd}}\right)$ in (30). This is due to the fact that the $\mathrm{BEC}$ droplets in the respective minima of the random potential can minimize their energy by deformation along the polarization axis [5]. The dipolar enhancement in both the quantum depletion $n^{\prime}$ and the external random potential depletion $n_{\mathrm{R}}$ are approximately the same with respect to $\epsilon_{\mathrm{dd}}$ up to $\epsilon_{\mathrm{dd}}=0.6$, but then the dipolar enhancement in $n_{\mathrm{R}}$ starts to increase faster than in $n^{\prime}$, see Fig. 1 .

Furthermore, we read off from (25) that the energy shift due to the interaction and the quantum fluctuations are ultraviolet divergent. Thus, following Refs. [43, 53], we can evaluate (25) by introducing an ultraviolet cutoff, where the interaction has to be renormalized by inserting the term $\frac{m\left(n V_{\mathbf{k}}\right)^{2}}{\hbar^{2} \mathbf{k}^{2}}$, so that the divergent part is removed

$$
\frac{E^{\prime}}{N}=\frac{1}{2 n} \int \frac{d^{3} \mathbf{k}}{(2 \pi)^{3}}\left[E_{\mathbf{k}}-\left(\frac{\hbar^{2} \mathbf{k}^{2}}{2 m}+n V_{\mathbf{k}}\right)+\frac{m\left(n V_{\mathbf{k}}\right)^{2}}{\hbar^{2} \mathbf{k}^{2}}\right],
$$

evaluations in the thermodynamic limit yielding

$$
\frac{E^{\prime}}{N}=\frac{2 \pi a \hbar^{2} n}{m} \frac{128}{15}\left(\frac{n a^{3}}{\pi}\right)^{\frac{1}{2}} Q_{\frac{5}{2}}\left(\epsilon_{\mathrm{dd}}\right)
$$

Another method for (25) relies on Schwinger trick [54] which leads again to (35). The energy shift due to the external random potential (27) is also ultraviolet divergent:

$$
\frac{E_{\mathrm{R}}}{N}=-\int \frac{d^{3} \mathbf{k}}{(2 \pi)^{3}} \frac{R_{0}}{\frac{\hbar^{2} \mathbf{k}^{2}}{2 m}+2 n V_{\mathbf{k}}} .
$$


Thus, it is calculated either with an ultraviolet cutoff by subtracting the divergent term $\frac{2 m R_{0}}{v \hbar^{2} \mathbf{k}^{2}}$, or with the help of the Schwinger trick, leading to

$$
\frac{E_{\mathrm{R}}}{N}=\frac{2 m R_{0}}{\hbar^{2}} \sqrt{\frac{n a}{\pi}} Q_{\frac{1}{2}}\left(\epsilon_{\mathrm{dd}}\right)
$$

Note that energy shift $E^{\prime}$ due to interaction and quantum fluctuations in equation (35) and the external random potential energy shift $E_{\mathrm{R}}$ in equation (37) reproduce the well-known contact interaction results when $\epsilon_{\mathrm{dd}}=0$, and that $E^{\prime}$ increases quicker than $E_{\mathrm{R}}$ with respect to $\epsilon_{\mathrm{dd}}$. Finally note that both energy shifts (35) and (37) could be used to study the collective properties of a trapped dipolar Bose gas within the local density approximation [55, 56.

\section{SUPERFLUIDITY}

Now we extend the Bogoliubov theory in order to study the transport phenomenon of superfluidity within the framework of linear response theory. With this we calculate the superfluid depletions due to both the external random potential and the thermal fluctuations.

\section{A. Bogoliubov theory revisited}

Following Refs. [34, 57] we calculate the superfluid component $n_{s}$ of the Bose gas, which moves with the superfluid velocity $\mathbf{v}_{\mathbf{s}}$, via a linear response approach. To this end we perform a Galilean boost for our system with a boost velocity $\mathbf{u}$ so that the normal fluid component $n_{n}$ is dragged along this boost. Inserting the Galilean transformations $\mathbf{x}^{\prime}=\mathbf{x}+\mathbf{u} t, t=t^{\prime}$, and rewriting the field operator in Heisenberg picture according to $\hat{\psi}^{\prime}\left(\mathbf{x}^{\prime}, t^{\prime}\right)=\hat{\psi}(\mathbf{x}, t) e^{\frac{2}{\hbar} m \mathbf{v}_{\mathbf{s}} \mathbf{x}}$, the Hamiltonian in momentum space reads instead of (4)

$$
\begin{aligned}
\hat{\mathcal{K}}= & \frac{1}{2} \sum_{\mathbf{k}}\left[\frac{\hbar^{2} \mathbf{k}^{2}}{2 m}-\mu_{\mathrm{eff}}\right]\left(\hat{a}_{\mathbf{k}}^{\dagger} \hat{a}_{\mathbf{k}}+\hat{a}_{-\mathbf{k}}^{\dagger} \hat{a}_{-\mathbf{k}}\right) \\
& +\frac{1}{2} \sum_{\mathbf{k}} \hbar \mathbf{k}\left(\mathbf{u}-\mathbf{v}_{\mathbf{s}}\right)\left(\hat{a}_{\mathbf{k}}^{\dagger} \hat{a}_{\mathbf{k}}-\hat{a}_{-\mathbf{k}}^{\dagger} \hat{a}_{-\mathbf{k}}\right) \\
& +\frac{1}{2 v} \sum_{\mathbf{p}, \mathbf{k}} U_{\mathbf{p}-\mathbf{k}}\left(\hat{a}_{\mathbf{p}}^{\dagger} \hat{a}_{\mathbf{k}}+\hat{a}_{-\mathbf{k}}^{\dagger} \hat{a}_{-\mathbf{p}}\right) \\
& +\frac{1}{2 v} \sum_{\mathbf{p}, \mathbf{k}, \mathbf{q}} V_{\mathbf{q}} \hat{a}_{\mathbf{k}+\mathbf{q}}^{\dagger} \hat{a}_{\mathbf{p}-\mathbf{q}}^{\dagger} \hat{a}_{\mathbf{p}} \hat{a}_{\mathbf{k}},
\end{aligned}
$$

with the effective chemical potential $\mu_{\mathrm{eff}}=\mu-\frac{1}{2} m \mathbf{v}_{\mathrm{s}}^{2}+$ $m \mathbf{u v}_{\mathrm{s}}$. Applying the Bogoliubov theory along similar lines as in Section II and after performing the disorder ensemble average of the grand-canonical Hamiltonian we obtain instead of (13)

$$
\begin{aligned}
\left\langle\hat{\mathcal{K}}^{\prime}\right\rangle= & v\left(-\mu_{\mathrm{eff}} n_{\mathbf{0}}+\frac{1}{2} V_{\mathbf{0}} n_{\mathbf{0}}^{2}\right) \\
& +\frac{1}{2} \sum_{\mathbf{k}}^{\prime}\left\{E_{\mathbf{k}}-\left[\frac{\hbar^{2} \mathbf{k}^{2}}{2 m}-\mu_{\mathrm{eff}}+n_{\mathbf{0}}\left(V_{\mathbf{0}}+V_{\mathbf{k}}\right)\right]\right\} \\
& +\sum_{\mathbf{k}}^{\prime}\left[E_{\mathbf{k}}+\hbar \mathbf{k}\left(\mathbf{u}-\mathbf{v}_{\mathrm{s}}\right)\right] \hat{\alpha}_{\mathbf{k}}^{\dagger} \hat{\alpha}_{\mathbf{k}} \\
& -\sum_{\mathbf{k}}^{\prime} \frac{n_{\mathbf{0}} R_{\mathbf{k}}\left(\frac{\hbar^{2} \mathbf{k}^{2}}{2 m}-\mu_{\mathrm{eff}}+n_{\mathbf{0}} V_{0}\right)}{\left\{E_{\mathbf{k}}^{2}-\left[\hbar \mathbf{k}\left(\mathbf{u}-\mathbf{v}_{\mathrm{s}}\right)\right]^{2}\right\}}
\end{aligned}
$$

where $E_{\mathbf{k}}$ stands for the quasi-particle dispersion (12) with $\mu$ substituted by $\mu_{\text {eff }}$. Now it is straightforward to find the corresponding grand-canonical effective potential, where we get instead of (14):

$$
\begin{aligned}
\Omega_{\mathrm{eff}}= & v\left(-\mu_{\mathrm{eff}} n_{\mathbf{0}}+\frac{1}{2} V_{\mathbf{0}} n_{\mathbf{0}}^{2}\right) \\
& +\frac{1}{2} \sum_{\mathbf{k}}^{\prime}\left\{E_{\mathbf{k}}-\left[\frac{\hbar^{2} \mathbf{k}^{2}}{2 m}-\mu_{\mathrm{eff}}+n_{\mathbf{0}}\left(V_{\mathbf{0}}+V_{\mathbf{k}}\right)\right]\right\} \\
& +\sum_{\mathbf{k}}^{\prime} \frac{1}{\beta} \ln \left\{1-e^{-\beta\left[E_{\mathbf{k}}+\hbar \mathbf{k}\left(\mathbf{u}-\mathbf{v}_{\mathrm{s}}\right)\right]}\right\} \\
& -\sum_{\mathbf{k}}^{\prime} \frac{n_{\mathbf{0}} R_{\mathbf{k}}\left(\frac{\hbar^{2} \mathbf{k}^{2}}{2 m}-\mu_{\mathrm{eff}}+n_{\mathbf{0}} V_{\mathbf{0}}\right)}{\left\{E_{\mathbf{k}}^{2}-\left[\hbar \mathbf{k}\left(\mathbf{u}-\mathbf{v}_{\mathrm{s}}\right)\right]^{2}\right\}}
\end{aligned}
$$

Within a linear response approach we are only interested in small velocities $\mathbf{v}_{\mathbf{S}}$ and $\mathbf{u}$, so we expand Eq. (40) up to second order in $\hbar \mathbf{k}\left(\mathbf{u}-\mathbf{v}_{\mathrm{S}}\right)$, and we get

$$
\begin{aligned}
\Omega_{\mathrm{eff}}= & v\left(-\mu_{\mathrm{eff}} n_{\mathbf{0}}+\frac{1}{2} V_{\mathbf{0}} n_{\mathbf{0}}^{2}\right) \\
& +\frac{1}{2} \sum_{\mathbf{k}}^{\prime}\left\{E_{\mathbf{k}}-\left[\frac{\hbar^{2} \mathbf{k}^{2}}{2 m}-\mu_{\mathrm{eff}}+n_{\mathbf{0}}\left(V_{\mathbf{0}}+V_{\mathbf{k}}\right)\right]\right\} \\
& +\sum_{\mathbf{k}}^{\prime} \frac{1}{\beta} \ln \left(1-e^{-\beta E_{\mathbf{k}}}\right)-\sum_{\mathbf{k}}^{\prime} \frac{\beta e^{\beta E_{\mathbf{k}}\left[\hbar \mathbf{k}\left(\mathbf{u}-\mathbf{v}_{\mathbf{s}}\right)\right]^{2}}}{2\left(e^{\beta E_{\mathbf{k}}}-1\right)^{2}} \\
& -\sum_{\mathbf{k}}^{\prime} \frac{n_{\mathbf{0}} R_{\mathbf{k}}}{E_{\mathbf{k}}^{2}}\left[\frac{\hbar^{2} \mathbf{k}^{2}}{2 m}-\mu_{\mathrm{eff}}+n_{\mathbf{0}} V_{\mathbf{0}}\right] \\
& -\sum_{\mathbf{k}}^{\prime} \frac{n_{\mathbf{0}} R_{\mathbf{k}}}{E_{\mathbf{k}}^{4}}\left[\frac{\hbar^{2} \mathbf{k}^{2}}{2 m}-\mu_{\mathrm{eff}}+n_{\mathbf{0}} V_{\mathbf{0}}\right]\left[\hbar \mathbf{k}\left(\mathbf{u}-\mathbf{v}_{\mathrm{s}}\right)\right]^{2} .
\end{aligned}
$$

Note that the terms linear in $\hbar \mathbf{k}\left(\mathbf{u}-\mathbf{v}_{\mathbf{s}}\right)$ vanish due to symmetry reasons. Extremizing the grand-canonical effective potential (41) with respect the the condensate density $n_{\mathbf{0}}$ yields in zeroth order the mean-field result $\mu_{\mathrm{eff}}=n_{\mathbf{0}} V_{\mathbf{0}}$. With this we are ready to determine the momentum of the system $\mathbf{P}=\left(-\frac{\partial \Omega_{\text {eff }}}{\partial \mathbf{u}}\right)_{v, T, \mu}$ in the smallvelocity limit, which turns out to be of the following form

$$
\mathbf{P}=m v\left(n \mathbf{v}_{s}+n_{\mathrm{n}} \mathbf{v}_{\mathrm{n}}\right),
$$


where the total density $n=n_{\mathrm{s}}+n_{\mathrm{n}}$ decomposes into the superfluid and normal component $n_{\mathrm{s}}$ and $n_{\mathrm{n}}$, respectively, and $\mathbf{v}_{\mathrm{n}}=\mathbf{u}-\mathbf{v}_{\mathrm{s}}$ denotes the normal fluid velocity. The normal fluid density turns out to decompose according to $n_{\mathrm{n}}=n_{\mathrm{R}}+n_{\mathrm{th}}$, where the contribution due to the external random potential, which only exists in the zero temperature limit, reads 37

$$
n_{\mathrm{R} i j}=\frac{1}{v} \sum_{\mathbf{k}}^{\prime} \frac{2 n R_{\mathbf{k}} \hbar^{2} k_{i} k_{j}}{m\left(\frac{\hbar^{2} \mathbf{k}^{2}}{2 m}\right)\left(\frac{\hbar^{2} \mathbf{k}^{2}}{2 m}+2 n V_{\mathbf{k}}\right)^{2}},
$$

while the normal fluid density due to the thermal fluctuations is given by

$$
n_{\mathrm{th} i j}=\frac{1}{v} \sum_{\mathbf{k}}^{\prime} \frac{\beta}{m} \hbar^{2} k_{i} k_{j} \frac{e^{\beta E_{\mathbf{k}}}}{\left(e^{\beta E_{\mathbf{k}}}-1\right)^{2}},
$$

where $E_{\mathbf{k}}$ stands for the Bogoliubov energy spectrum (23). Note that there is no superfluid depletion in (42) which is due to quantum fluctuations, in contrast to the condensate depletion which has a quantum fluctuations component determined by Eq. (20).

\section{B. Zero-temperature superfluid depletion}

Again we specialize at first to the zero-temperature limit and to a delta-correlated random potential and evaluate the corresponding superfluid depletions in the thermodynamic limit. Depending on the boost direction, we have two different superfluid depletions in the directions parallel or perpendicular to the dipole polarization. In the first case the superfluid depletion reads

$$
n_{\mathrm{R} \|}=4 n_{\mathrm{HM}} J_{-\frac{1}{2}}\left(\epsilon_{\mathrm{dd}}\right) \text {, }
$$

whereas in the second case the superfluid depletion turns out to be

$$
n_{\mathrm{R} \perp}=2 n_{\mathrm{HM}}\left[Q_{-\frac{1}{2}}\left(\epsilon_{\mathrm{dd}}\right)-J_{-\frac{1}{2}}\left(\epsilon_{\mathrm{dd}}\right)\right] .
$$

Here we have introduced the new function

$$
J_{\alpha}\left(\epsilon_{\mathrm{dd}}\right)=\int_{0}^{1} x^{2}\left[1+\epsilon_{\mathrm{dd}}\left(3 x^{2}-1\right)\right]^{\alpha} d x,
$$

which can also be expressed analytically with the help of the integral table [52] according to

$$
J_{\alpha}\left(\epsilon_{\mathrm{dd}}\right)=\frac{1}{3}\left(1-\epsilon_{\mathrm{dd}}\right)^{\alpha}{ }_{2} F_{1}\left(-\alpha, \frac{3}{2} ; \frac{5}{2} ; \frac{-3 \epsilon_{\mathrm{dd}}}{1-\epsilon_{\mathrm{dd}}}\right) .
$$

Note that the function $J_{\alpha}\left(\epsilon_{\mathrm{dd}}\right)$ shown in Fig. 2 has the property $J_{\alpha}(0)=1 / 3$ and decreases slowly with increasing $\epsilon_{\mathrm{dd}}$ for $\alpha=-\frac{1}{2}$, while it behaves non-monotonically with increasing $\epsilon_{\mathrm{dd}}$ for $\alpha=-\frac{5}{2}$.

We observe that, due to $Q_{\alpha}(0)=1$ and $J_{\alpha}(0)=1 / 3$, both depletions $n_{\mathrm{R} \|}$ and $n_{\mathrm{R} \perp}$ coincide for vanishing dipolar interaction and equate $\frac{4}{3} n_{\mathrm{HM}}$, which reproduces the

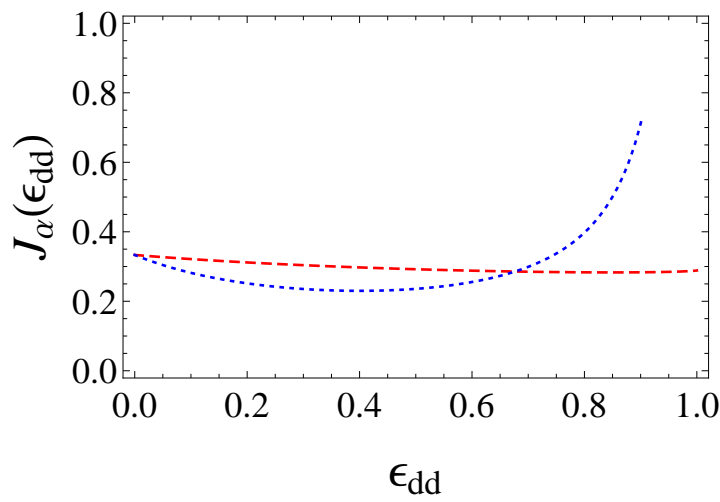

Figure 2: (color online). Function $J_{\alpha}\left(\epsilon_{\mathrm{dd}}\right)$ versus relative dipolar interaction strength $\epsilon_{\mathrm{dd}}$ for different values of $\alpha$ : $1 / 2$ (red, dashed), $-5 / 2$ (blue, dotted).

Huang-Meng result for the isotropic contact interaction [31]. Due to the localization of bosons in the respective minima of the random potential the superfluid is hampered, so that the superfluid depletion is larger than the condensate depletion. Switching on the dipolar interaction has a significant effect on both depletions $n_{\mathrm{R} \|}$ and $n_{\mathrm{R} \perp}$. Their ratio with the condensate depletion $n_{\mathrm{R}}$ turns out to be monotonically decreasing/increasing with respect to the relative dipolar interaction strength $\epsilon_{\mathrm{dd}}$ according to Fig. 3. This is explained qualitatively by considering the dipolar interaction in Fourier space (5) as an effective contact interaction [37, 39]. Therefore, for sufficiently large values of $\epsilon_{\mathrm{dd}}$, it turns out that $n_{\mathrm{R} \|}$ becomes even smaller than the condensate depletion. This surprising finding suggests that the localization of the bosons in the minima of the random potential only occurs for a finite period of time [58]. As a consequence the ratio $n_{\mathrm{R} \|} / n_{\mathrm{R} \perp}$ decreases monotonically for increasing values of the relative dipolar interaction strength $\epsilon_{\mathrm{dd}}$.

\section{FINITE-TEMPERATURE EFFECTS}

In the previous sections we restricted the application of the Bogoliubov theory of dirty bosons to the zerotemperature limit. Nevertheless any experiment is performed at finite-temperature, where thermal fluctuations play an important role besides quantum and disorder fluctuations. Therefore, we calculate in this section for a dipolar BEC the contributions to both the condensate and the normal fluid component which are due to thermal excitations.

\section{A. Condensate depletion}

At zero temperature the condensate depletion consists of two contributions, one due to the quantum fluctuation (20) and another one due to the disorder potential (22), 

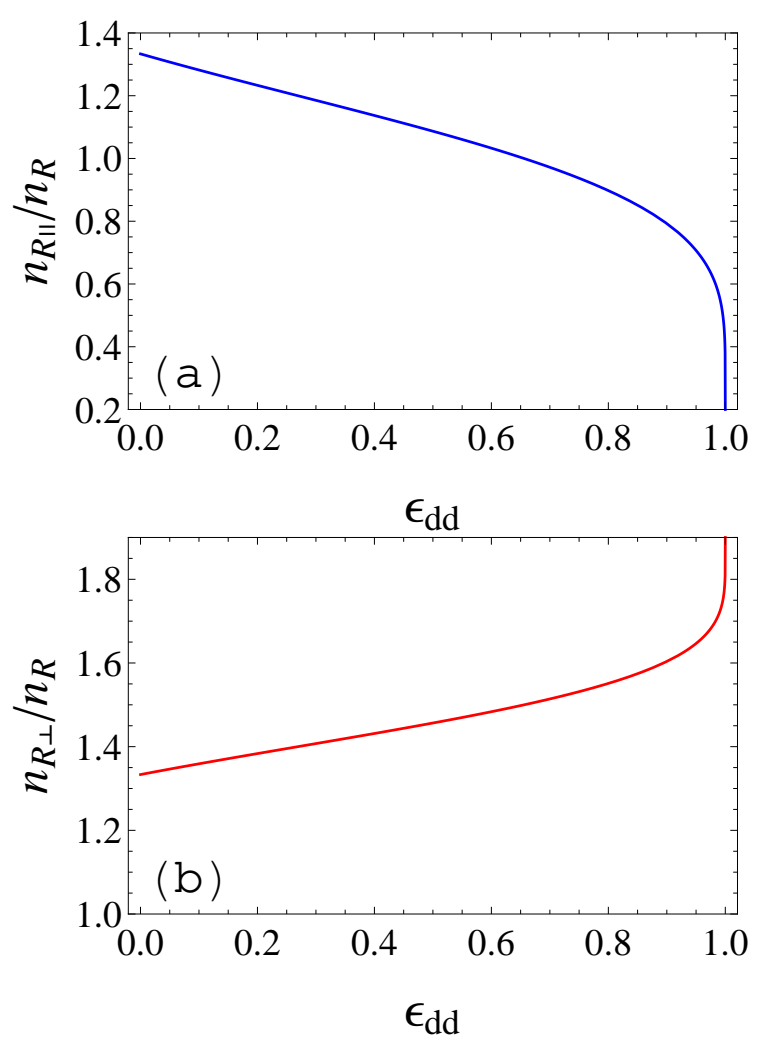

Figure 3: (color online). Ratios of superfluid depletions $n_{\mathrm{R} \|}$ and $n_{\mathrm{R} \perp}$ and condensate depletion $n_{\mathrm{R}}$ versus relative dipolar interaction strength $\epsilon_{\mathrm{dd}}$.

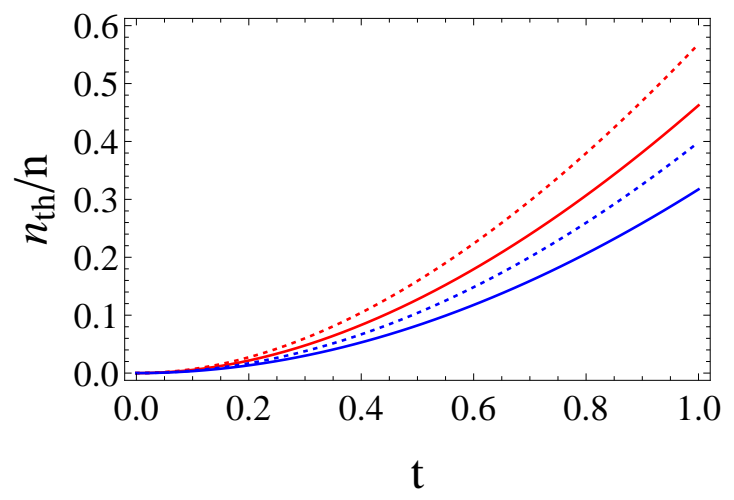

Figure 4: (color online). Thermal fractional depletion $n_{\mathrm{th}} / n$ versus relative temperature $t=T / T_{c}^{0}$ for different values of relative dipolar interaction strength $\epsilon_{\mathrm{dd}}=0$ (solid), $\epsilon_{\mathrm{dd}}=0.8$ (dotted), and gas parameter $\gamma=0.01$ (red, upper two curves), $\gamma=0.20$ (blue).

which are evaluated in (28), (30) for a dipolar BEC, respectively. But for increasing temperature also the third contribution (21) of the depletion (19) will play an important role and should be taken into account. This additional term represents the condensate depletion due to the thermal excitations, which reads in the thermodynamics limit

$$
n_{\mathrm{th}}=\int \frac{d^{3} \mathbf{k}}{(2 \pi)^{3}} \frac{\frac{\hbar^{2} \mathbf{k}^{2}}{2 m}+n V_{\mathbf{k}}}{E_{\mathbf{k}}} \frac{1}{e^{\beta E_{\mathbf{k}}-1}},
$$

where $E_{\mathbf{k}}$ denotes the Bogoliubov spectrum (23). The integral (49) can be recast in dimensionless form

$$
\frac{n_{\mathrm{th}}}{n}=\frac{\gamma^{-\frac{1}{6}} t^{2}}{2 \pi^{\frac{1}{2}}\left(\zeta\left(\frac{3}{2}\right)\right)^{\frac{4}{3}}} I\left(\gamma, \epsilon_{\mathrm{dd}}, t\right)
$$

where we have introduced the gas parameter $\gamma=n a^{3}$, and the relative temperature $t=T / T_{c}^{0}$, with $T_{c}^{0}=$ $\frac{2 \pi \hbar^{2} n^{\frac{2}{3}}}{\left(\zeta\left(\frac{3}{2}\right)\right)^{\frac{2}{3}} m k_{\mathrm{B}}}$ being the critical temperature for the noninteracting Bose gas, and $\zeta(x)$ denotes the Riemann zeta function with the value $\zeta\left(\frac{3}{2}\right)=2.61238 \ldots$ The remaining integral $I\left(\gamma, \epsilon_{\mathrm{dd}}, t\right)$ reads

$$
I\left(\gamma, \epsilon_{\mathrm{dd}}, t\right)=\int_{0}^{\infty} d x \int_{0}^{\pi} d \theta \frac{x \sin \theta\left(1+\frac{\alpha x^{2}}{8 \Theta^{2}}\right)}{\sqrt{\Theta+\frac{\alpha x^{2}}{16 \Theta}}\left(e^{\sqrt{x^{2}+\frac{\alpha x^{4}}{16 \Theta^{2}}}}-1\right)},
$$

with the abbreviations $\alpha=\left[\frac{t}{\gamma^{\frac{1}{3}}\left(\zeta\left(\frac{3}{2}\right)\right)^{\frac{2}{3}}}\right]^{2}$ and $\Theta=$ $1+\epsilon_{\mathrm{dd}}\left(3 \cos ^{2} \theta-1\right)$. Fig. 4 shows how the condensate thermal fractional depletion (50) increases with the relative temperature $t$ for different values of the relative dipolar interaction strength $\epsilon_{\mathrm{dd}}$ and the gas parameter $\gamma$. For increasing contact interaction, it turns out that $n_{\text {th }}$ decreases as collisions between the thermal particles yield partially a scattering into the macroscopic ground state. In contrast to that we observe that the dipolar interaction has the opposite effect: increasing $\epsilon_{\mathrm{dd}}$ enhances the thermal depletion $n_{\mathrm{th}}$. This can be understood from considering the consequences of the dipolar interaction as an effective contact interaction [37, 39], yielding two attractive and one repulsive direction in Fourier space according to (5).

In order to investigate this qualitative finding more quantitatively in the vicinity of zero temperature but far below the critical temperature, we approximate the condensate depletion (50) analytically like

$$
\begin{aligned}
\frac{n_{\mathrm{th}}}{n}= & \frac{\pi^{\frac{3}{2}} \gamma^{-\frac{1}{6}} t^{2}}{6\left(\zeta\left(\frac{3}{2}\right)\right)^{\frac{4}{3}}} Q_{-\frac{1}{2}}\left(\epsilon_{\mathrm{dd}}\right) \\
& -\frac{\pi^{\frac{7}{2}} \gamma^{-\frac{5}{6}} t^{4}}{480\left(\zeta\left(\frac{3}{2}\right)\right)^{\frac{8}{3}}} Q_{-\frac{5}{2}}\left(\epsilon_{\mathrm{dd}}\right)+\ldots .
\end{aligned}
$$

This reproduces the isotropic contact interaction case for vanishing dipolar interaction due to $Q_{\alpha}(0)=1$ [59]. Note that (52) turns out to approximate (51) quite well irrespective of $\gamma$ and $\epsilon_{\mathrm{dd}}$ in the temperature range $0 \leq t \leq$ 0.3 . Here also each term in (52) decreases for increasing $\gamma$, while it is enhanced for increasing $\epsilon_{\mathrm{dd}}$. 

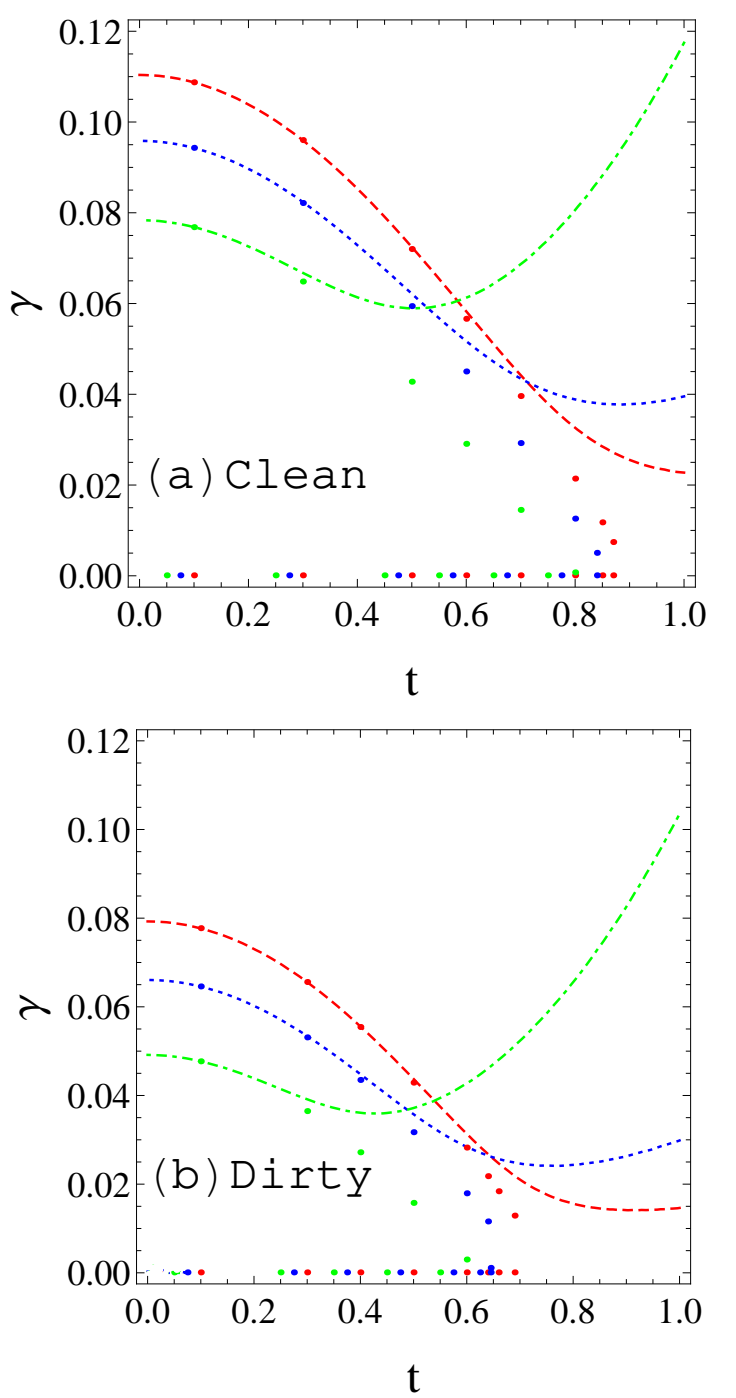

Figure 5: (color online). Validity range of Bogoliubov theory in the $t-\gamma$ plane for (a) clean case, i.e. $R_{0}=0$, and (b) dirty case with $R_{0}=\frac{2 \hbar^{4} \pi^{\frac{3}{2}} n^{\frac{1}{3}}}{5 m^{2}}$ for different values of relative dipolar interaction strength $\epsilon_{\mathrm{dd}}=0$ (red, dashed), $\epsilon_{\mathrm{dd}}=0.5$ (blue, dotted), $\epsilon_{\mathrm{dd}}=0.8$ (green, dotted-dashed). Condensate depletion (53) equals $n / 2$ for curves with $n_{\text {th }} / n$ from (52). Dots represent the case upon inserting $n_{\mathrm{th}} / n$ from (50), (51).

Now we investigate how the validity range of the Bogoliubov theory depends on the gas parameter $\gamma$ and the relative temperature $t$ for different values of the relative dipolar interaction strength $\epsilon_{\mathrm{dd}}$ and the disorder strength $R_{0}$. To this end we restrict the condensate depletion $\Delta n=n-n_{\mathbf{0}}$ in equation (19) to be limited maximally by half of the particle number density $n$. Inserting the critical temperature for the non-interacting Bose gas $T_{c}^{0}$ and the gas parameter $\gamma$ in the condensate depletion due to quantum fluctuation (28) and random potential (30), the fractional depletion reads

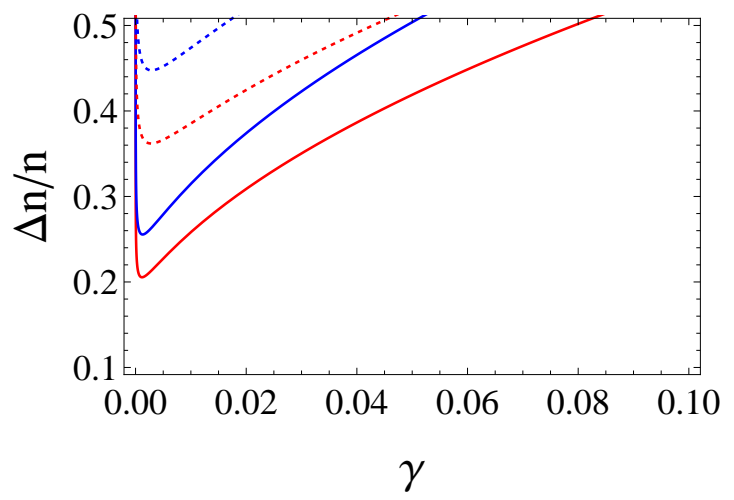

Figure 6: (color online). Fractional depletion $\Delta n / n$ versus gas parameter $\gamma=n a^{3}$ for different values of relative dipolar interaction strength $\epsilon_{\mathrm{dd}}=0$ (red), $\epsilon_{\mathrm{dd}}=0.8$ (blue) and relative temperature $t=0$ (solid), $t=0.5$ (dotted) with the disorder strength $R_{0}=\frac{2 \hbar^{4} \pi^{\frac{3}{2}} n^{\frac{1}{3}}}{5 m^{2}}$.

$$
\begin{aligned}
\frac{\Delta n}{n}= & \frac{8}{3 \sqrt{\pi}} \gamma^{\frac{1}{2}} Q_{\frac{3}{2}}\left(\epsilon_{\mathrm{dd}}\right)+\frac{n_{\mathrm{th}}}{n} \\
& +\frac{m^{2} R_{0}}{8 \hbar^{4} \pi^{\frac{3}{2}} n^{\frac{1}{3}}} \gamma^{-\frac{1}{6}} Q_{-\frac{1}{2}}\left(\epsilon_{\mathrm{dd}}\right) .
\end{aligned}
$$

Here the thermal fractional condensate depletion (50) in (53) is determined numerically from (51) and from (52) analytically in the vicinity of zero temperature for comparison. Fig. 5 depicts the resulting validity range of the Bogoliubov theory in the clean case i.e. the disorder strength $R_{0}=0$, and in the dirty case with $R_{0}=\frac{2 \hbar^{4} \pi^{\frac{3}{2}} n^{\frac{1}{3}}}{5 m^{2}}$. It is represented by the area below the curves when the condensate depletion is equal to $n / 2$. Outside this area the Bogoliubov approximation is not valid. Note that the validity range (50), (53) is well approximated by (52) near zero temperature. From (50), (53) we read off that the Bogoliubov theory is not applicable near the critical temperature. Furthermore the validity range decreases for increasing values of the relative dipolar interaction strength $\epsilon_{\mathrm{dd}}$, and it also smaller in the dirty case compared to the clean one.

Finally, we plot in Fig. 6 the total fractional condensate depletion $\frac{\Delta n}{n}$ versus the gas parameter $\gamma$ with the thermal fractional condensate depletion $\frac{n_{\text {th }}}{n}$ in (53) inserted from (50) for the disorder strength $R_{0}=\frac{2 \hbar^{4} \pi^{\frac{3}{2}} n^{\frac{1}{3}}}{5 m^{2}}$. Note that the sharp increase for small gas parameter is unphysical due to the fact that the Bogoliubov theory is no longer valid, see Fig. 15. We conclude that increasing the parameters $t$ and $\epsilon_{\mathrm{dd}}$ yields an increasing fractional depletion.

\section{B. Superfluid depletion}

Now we calculate the superfluid depletion for the dipolar Bose gas which is due to thermal fluctuations. In the 

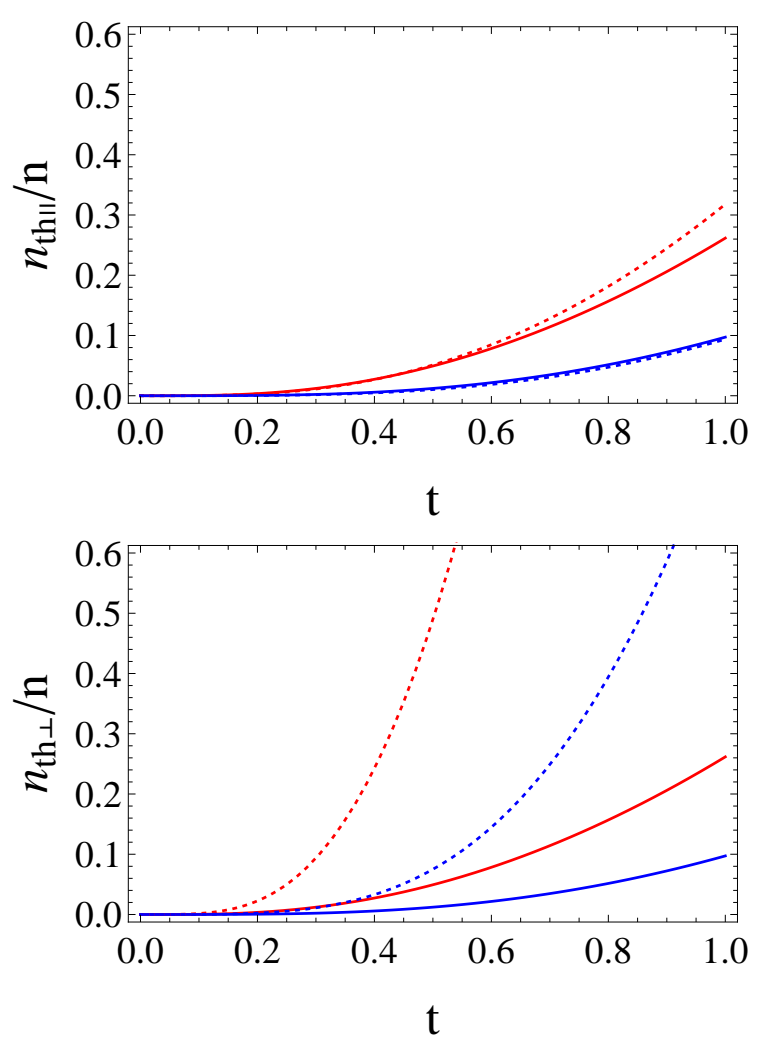

Figure 7: (color online). Superfluid thermal fractional depletions $n_{\mathrm{th} \|} / n$ and $n_{\mathrm{th} \perp} / n$ versus relative temperature $t=T / T_{c}^{0}$ for different values of relative dipolar interaction strength $\epsilon_{\mathrm{dd}}=0$ (solid), $\epsilon_{\mathrm{dd}}=0.6$ (dotted) and gas parameter $\gamma=0.01$ (red), $\gamma=0.20$ (blue).

thermodynamic limit of equation (44) the components parallel and perpendicular to the dipole polarization direction have to be evaluated separately. Parallel to the dipoles the integral reads in dimensionless form

$$
\frac{n_{\mathrm{th} \|}}{n}=\frac{\gamma^{-\frac{5}{6}} t^{4}}{8 \pi^{\frac{1}{2}}\left(\zeta\left(\frac{3}{2}\right)\right)^{\frac{8}{3}}} I_{\|}\left(\gamma, \epsilon_{\mathrm{dd}}, t\right),
$$

where the remaining integral $I_{\|}\left(\gamma, \epsilon_{\mathrm{dd}}, t\right)$ is written explicitly as

$$
I_{\|}\left(\gamma, \epsilon_{\mathrm{dd}}, t\right)=\int_{0}^{\infty} d x \int_{0}^{\pi} d \theta \frac{x^{4} \sin \theta \cos ^{2} \theta e^{\sqrt{x^{2}+\frac{\alpha x^{4}}{16 \Theta^{2}}}}}{\Theta^{\frac{5}{2}}\left(e^{\sqrt{x^{2}+\frac{\alpha x^{4}}{16 \Theta^{2}}}}-1\right)^{2}}
$$

In the direction perpendicular to the dipoles the superfluid depletion reads in dimensionless form

$$
\frac{n_{\mathrm{th} \perp}}{n}=\frac{\gamma^{-\frac{5}{6}} t^{4}}{8 \pi^{\frac{1}{2}}\left(\zeta\left(\frac{3}{2}\right)\right)^{\frac{8}{3}}} I_{\perp}\left(\gamma, \epsilon_{\mathrm{dd}}, t\right)
$$
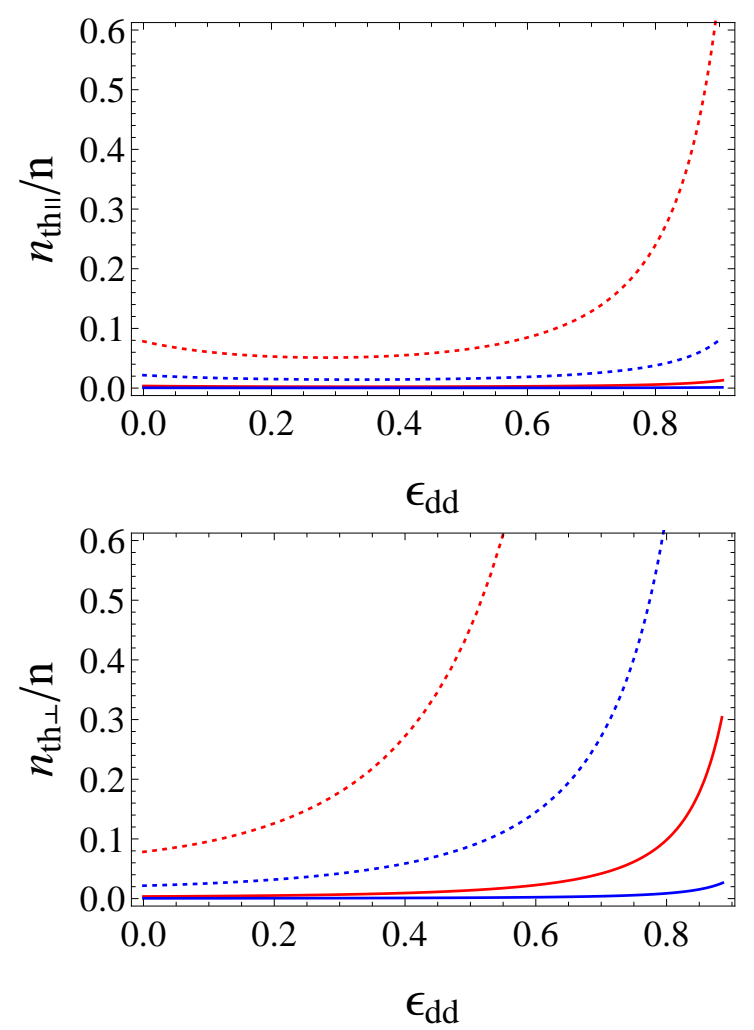

Figure 8: (color online). Superfluid thermal fractional depletions $n_{\mathrm{th} \|} / n$ and $n_{\mathrm{th} \perp} / n$ versus relative dipolar interaction strength $\epsilon_{\mathrm{dd}}$ for different values of relative temperature $t=0.2$ (solid), $t=0.6$ (dotted) and gas parameter $\gamma=0.01$ (red), $\gamma=0.20$ (blue).

where the remaining integral $I_{\perp}\left(\gamma, \epsilon_{\mathrm{dd}}, t\right)$ is written explicitly as

$$
I_{\perp}\left(\gamma, \epsilon_{\mathrm{dd}}, t\right)=\int_{0}^{\infty} d x \int_{0}^{\pi} d \theta \frac{x^{4} \sin ^{3} \theta e^{\sqrt{x^{2}+\frac{\alpha x^{4}}{16 \Theta^{2}}}}}{2 \Theta^{\frac{5}{2}}\left(e^{\sqrt{x^{2}+\frac{\alpha x^{4}}{16 \Theta^{2}}}}-1\right)^{2}} .
$$

Fig. 7 shows how the superfluid thermal fractional depletions parallel and perpendicular to the polarized dipoles depend on the respective values of the parameters $\gamma, \epsilon_{\mathrm{dd}}$,

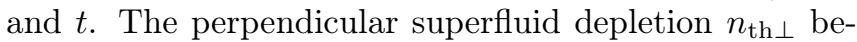
haves similar to the condensate thermal depletion $n_{\mathrm{th}}$, i.e. $n_{\mathrm{th} \perp}$ is enhanced for increasing $t$ and $\epsilon_{\mathrm{dd}}$, whereas it is reduced for increasing $\gamma$. Also for $n_{\mathrm{th} \|}$ we observe

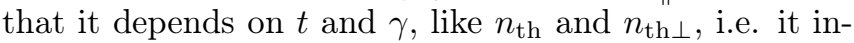
creases with $t$ but decreases with $\gamma$. But, surprisingly, it turns out that $n_{\mathrm{th} \|}$ has a nontrivial dependence on $\epsilon_{\mathrm{dd}}$, where we have first a decrease and then an increase for increasing $\epsilon_{\mathrm{dd}}$. Fig. 8 highlights in more detail how the superfluid thermal fractional depletions changes versus relative dipolar interaction strength $\epsilon_{\mathrm{dd}}$.

Note that both $n_{\mathrm{th} \perp}$ and $n_{\mathrm{th} \|}$ coincide for vanishing dipolar interaction as follows from Fig. 9 which shows the thermal superfluid depletion ratio $n_{\mathrm{th} \|} / n_{\mathrm{th} \perp}$ versus 


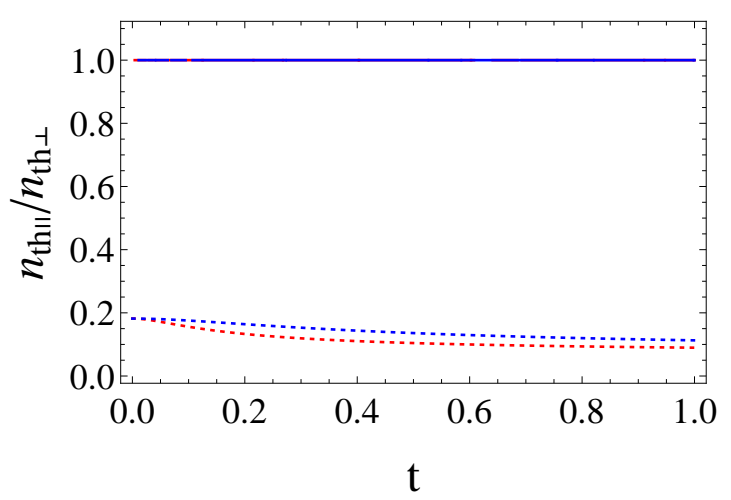

Figure 9: (color online). Ratios of thermal superfluid depletions $n_{\mathrm{th} \|} / n_{\mathrm{th} \perp}$ versus relative temperature $t=T / T_{c}^{0}$ for different values of relative dipolar strength $\epsilon_{\mathrm{dd}}=0$ (solid), $\epsilon_{\mathrm{dd}}=0.6$ (dotted) and gas parameter $\gamma=0.01$ (red), $\gamma=0.20$ (blue).

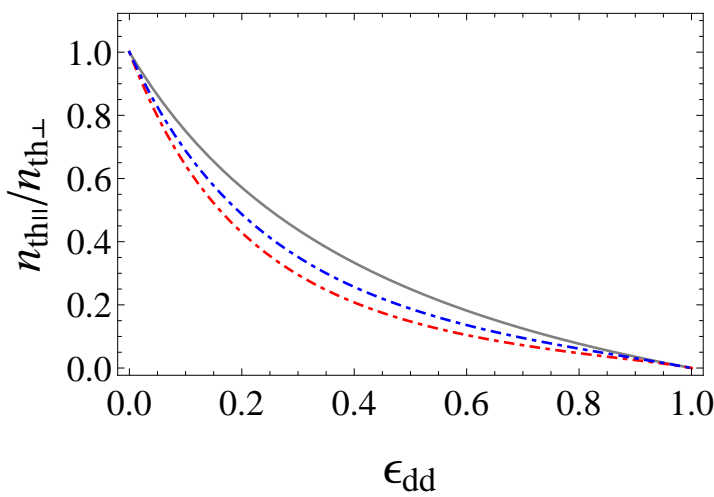

Figure 10: (color online). Ratios of thermal superfluid depletions $n_{\mathrm{th} \|} / n_{\mathrm{th} \perp}$ versus relative dipolar interaction strength $\epsilon_{\mathrm{dd}}$ for different values of the gas parameter $\gamma=0.01$ (red), $\gamma=0.20$ (blue) and relative temperature $t=0$ (solid-gray), $t=0.5$ (dotted-dashed) with $t=0$ limit from (58), (59).

the relative temperature $t$. When the dipolar interaction is present we notice that the ratio $n_{\mathrm{th} \|} / n_{\mathrm{th} \perp}$ reveals only a tiny $t$ - and $\gamma$-dependence which is decreasing with $t$ and increasing with $\gamma$, respectively. In Fig. 10] we plot the thermal superfluid depletions ratio $n_{\mathrm{th} \|} / n_{\mathrm{th} \perp}$ versus the relative dipolar strength $\epsilon_{\mathrm{dd}}$. It shows that both $n_{\mathrm{th} \perp}$ and $n_{\mathrm{th} \|}$ coincide for vanishing dipolar interaction, and the ratio $n_{\mathrm{th} \|} / n_{\mathrm{th} \perp}$ decreases for increasing values of the relative dipolar strength $\epsilon_{\mathrm{dd}}$.

In the vicinity of the zero temperature but far below critical temperature we can approximate the superfluid depletion in (54) analytically. We find that the superfluid depletion parallel to the dipoles reads

$$
\frac{n_{\mathrm{th} \|}}{n}=\frac{\pi^{\frac{7}{2}} \gamma^{-\frac{5}{6}} t^{4}}{15\left(\zeta\left(\frac{3}{2}\right)\right)^{\frac{8}{3}}} J_{-\frac{5}{2}}\left(\epsilon_{\mathrm{dd}}\right)+\ldots
$$

which has a non-monotonic behavior versus $\epsilon_{\mathrm{dd}}$ due to the function $J_{-\frac{5}{2}}\left(\epsilon_{\mathrm{dd}}\right)$, which is plotted in Fig. 2 where we have a decrease at first and then an increase for increasing
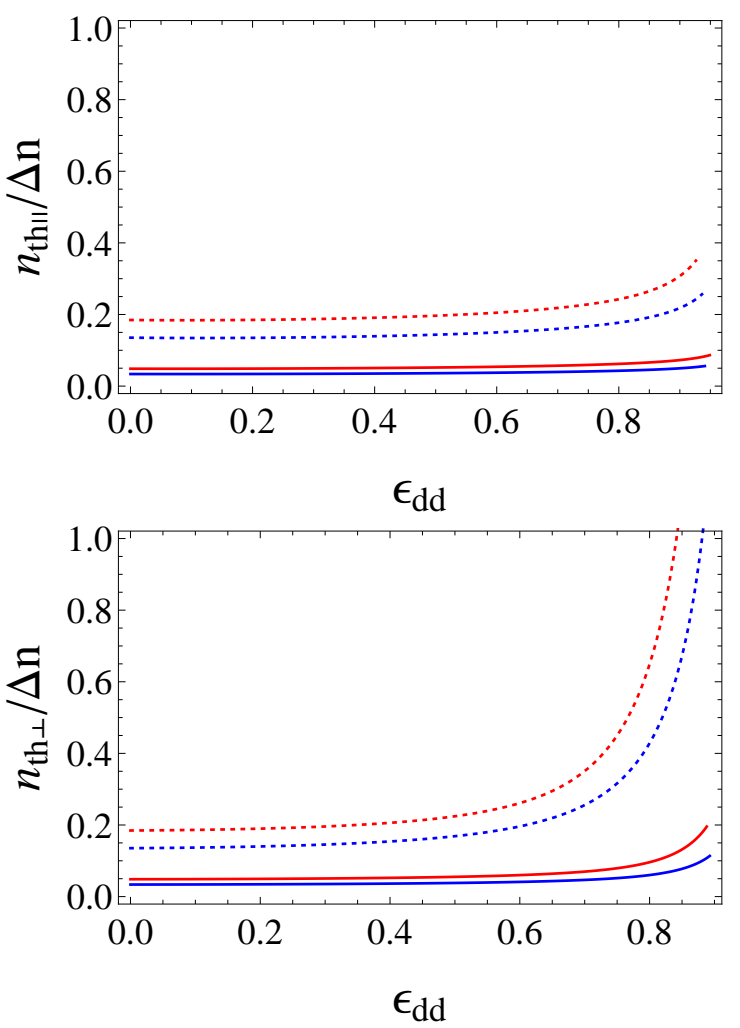

Figure 11: (color online). Ratios of superfluid and condensate depletions $n_{\mathrm{th} \|} / \Delta n$ (upper) and $n_{\mathrm{th} \perp} / \Delta n$ (lower) versus relative dipolar strength $\epsilon_{\mathrm{dd}}$ for different values of relative temperature $t=0.3$ (solid), $t=0.6$ (dotted) and gas parameter $\gamma=0.01$ (red), $\gamma=0.11$ (blue) in the clean case $R_{0}=0$.

$\epsilon_{\mathrm{dd}}$. Furthermore, the superfluid depletion perpendicular to the dipoles reads

$$
\frac{n_{\mathrm{th} \perp}}{n}=\frac{\pi^{\frac{7}{2}} \gamma^{-\frac{5}{6}} t^{4}}{30\left(\zeta\left(\frac{3}{2}\right)\right)^{\frac{8}{3}}}\left[Q_{-\frac{5}{2}}\left(\epsilon_{\mathrm{dd}}\right)-J_{-\frac{5}{2}}\left(\epsilon_{\mathrm{dd}}\right)\right]+\ldots
$$

so that $n_{\mathrm{th} \perp}$ is enhanced for increasing $\epsilon_{\mathrm{dd}}$ due to the fact that the function $Q_{-\frac{5}{2}}\left(\epsilon_{\mathrm{dd}}\right)$ shown in Fig. 11increases faster than the function $J_{-\frac{5}{2}}\left(\epsilon_{\mathrm{dd}}\right)$ shown in Fig. 22. A comparison between the two different superfluid depletions shows that both $n_{\mathrm{th} \perp}$ and $n_{\mathrm{th} \|}$ coincide for vanishing dipolar interaction, and reproduce the isotropic contact interaction result [59] due to $Q_{\alpha}(0)=1$ and $J_{\alpha}(0)=1 / 3$. The ratio $n_{\mathrm{th} \|} / n_{\mathrm{th} \perp}$ from (58), (59) decreases for increasing values of the relative dipolar strength $\epsilon_{\mathrm{dd}}$ as is shown in gray color in Fig. 10.

\section{Superfluid Ratios}

Finally after having discussed the different origins of the superfluid depletions for the dipolar Bose gas, we discuss now the ratios of the total superfluid depletions over the total condensate depletion $\Delta n$ for different values of the respective parameters $\gamma, \epsilon_{\mathrm{dd}}$, and $t$. We consider both 

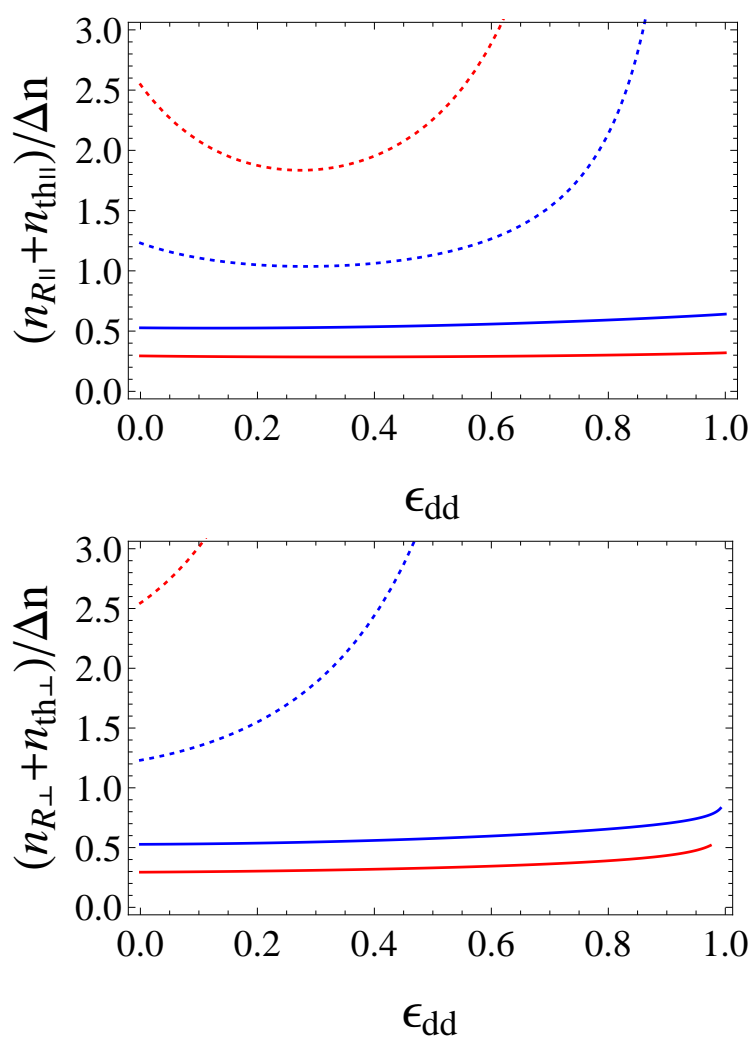

Figure 12: (color online). Ratios of superfluid depletions $\left(n_{\mathrm{th} \|}+n_{\mathrm{R} \|}\right) / \Delta n$ (upper) and $\left(n_{\mathrm{th} \perp}+n_{\mathrm{R} \perp}\right) / \Delta n$ (lower) versus relative dipolar strength $\epsilon_{\mathrm{dd}}$ for different values of relative temperature $t=0$ (solid), $t=0.5$ (dotted) and gas parameter $\gamma=0.01$ (red), $\gamma=0.08$ (blue) in the dirty case $R_{0}=\frac{2 \hbar^{4} \pi^{\frac{3}{2}} n^{\frac{1}{3}}}{5 m^{2}}$.

the clean case where the disorder strength $R_{0}=0$ and in the dirty case with $R_{0}=\frac{2 \hbar^{4} \pi^{\frac{3}{2}} n^{\frac{1}{3}}}{5 m^{2}}$. Fig. 11] shows the clean case with the ratios $n_{\mathrm{th} \|} / \Delta n$ (upper) and $n_{\mathrm{th} \perp} / \Delta n$ (lower) versus relative dipolar strength $\epsilon_{\mathrm{dd}}$. For increasing $\epsilon_{\mathrm{dd}}$ and $t$, both $n_{\mathrm{th} \|} / \Delta n$ and $n_{\mathrm{th} \perp} / \Delta n$ are enhanced, but increasing $\gamma$ decreases both $n_{\mathrm{th} \|} / \Delta n$ and $n_{\mathrm{th} \perp} / \Delta n$.

Fig. 12 shows in the dirty case the ratios $\left(n_{\mathrm{th} \|}+\right.$ $\left.n_{\mathrm{R} \|}\right) / \Delta n$ (upper) and $\left(n_{\mathrm{th} \perp}+n_{\mathrm{R} \perp}\right) / \Delta n$ (lower) versus relative dipolar strength $\epsilon_{\mathrm{dd}}$. For increasing $\epsilon_{\mathrm{dd}}$ we will get enhancement for $\left(n_{\mathrm{th} \perp}+n_{\mathrm{R} \perp}\right) / \Delta n$, regardless of the temperature $t$, while for $\left(n_{\mathrm{th} \|}+n_{\mathrm{R} \|}\right) / \Delta n$ we will get enhancement for small values of $t$ and then upon increasing $t$, we observe a decrease up to $\epsilon_{\mathrm{dd}}=0.3$ and then an increase, which shows that the disorder changes the situation a lot compared to the clean case in Fig. 11] Increasing $\gamma$ will cause a more complex interplay on both $\left(n_{\mathrm{th} \|}+n_{\mathrm{R} \|}\right) / \Delta n$ and $\left(n_{\mathrm{th} \perp}+n_{\mathrm{R} \perp}\right) / \Delta n$, as is seen in Fig. 12,

\section{CONCLUSION}

In this paper we have investigated the dipolar dirty boson problem, where both the short-range isotropic contact interaction as well as the anisotropic longrange dipole-dipole interaction are present within a Bogoliubov theory at low temperatures below the critical temperature. In the zero-temperature limit, we discussed analytically the nontrivial results for both the condensate and the superfluid depletions as well as the interplay between the long-range anisotropic dipolar interaction and the external random potential which yields an anisotropic superfluidity [37, 39]. At finite temperature, we obtained, due to the long-range anisotropic dipole-dipole interaction, different analytical and numerical results for condensate and the superfluid depletions, where the latter depends sensitively on whether the flow direction is parallel or perpendicular to the dipolar polarization axis. It still remains to investigate how the Josephson relation between condensate and superfluid densities reads for a disordered dipolar superfluid [60].

\section{ACKNOWLEDGMENT}

We both acknowledge fruitful discussions with $T$. Checinski and A. R. P. Lima. M. Ghabour thanks his family for financial support during this work. A. Pelster thanks for the support from the German Research Foundation (DFG) via the Collaborative Research Center SFB / TR49 Condensed Matter Systems with Variable Many-Body Interactions.
[1] J. Stenger, D. M. Stamper-Kurn, M. R. Andrews, A. P. Chikkatur, S. Inouye, H. J. Miesner, and W. Ketterle. J. Low Temp. Phys. 113, 167 (1998).

[2] F. Dalfovo, S. Giorgini, L. P. Pitaevskii, and Sandro Stringari. Rev. Mod. Phys. 71, 463 (1999).

[3] A. J. Leggett. Rev. Mod. Phys. 73, 307 (2001).

[4] A. Griesmaier, J. Werner, S. Hensler, J. Stuhler, and T. Pfau. Phys. Rev. Lett. 94, 160401 (2005).

[5] T. Lahaye, T. Koch, B. Frohlich, M. Fattori, J. Metz, A. Griesmaier, S. Giovanazzi, and T. Pfau. Nature 448, 672
(2007).

[6] M. Lu, N. Q. Burdick, S. H. Youn, and B. L. Lev. Phys. Rev. Lett. 107, 190401 (2011).

[7] K. Aikawa, A. Frisch, M. Mark, S. Baier, A. Rietzler, R. Grimm, and F. Ferlaino. Phys. Rev. Lett. 108, 210401 (2012).

[8] S. Ospelkaus, K. K. Ni, M. H. G. de Miranda, B. Neyenhuis, D. Wang, S. Kotochigova, P. S. Julienne, D. S. Jin, and J. Ye. Faraday Discuss. 142, 351 (2009).

[9] S. Ospelkaus, K. K. Ni, G. Quèmèner, B. Neyenhuis, D. 
Wang, M. H. G. de Miranda, J. L. Bohn, J. Ye, and D. S. Jin. Phys. Rev. Lett. 104, 030402 (2010).

[10] R. Feynman. Int. J. Theo. Phys. 21, 467 (1982).

[11] Y. J. Lin, R. L. Compton, K. Jiménez-García, J. V. Porto, and I. B. Spielman. Nature 462, 628 (2009).

[12] Y. J. Lin, K. Jiménez-García, and I. B. Spielman. Nature 471, 83 (2011).

[13] H. J. Metcalf and P. van der Straten, Laser Cooling and Trapping (Springer, Berlin, 1999).

[14] A. L. Gaunt, T. F. Schmidutz, I. Gotlibovych, R. P. Smith, and Z. Hadzibabic. Phys. Rev. Lett. 110, 200406 (2013).

[15] V. Bretin, S. Stock, Y. Seurin, and Jean Dalibard. Phys. Rev. Lett. 92, 050403 (2004).

[16] S. Inouye, M. R. Andrews, J. Stenger, H. J. Miesner, D. M. Stamper-Kurn, and W. Ketterle. Nature 392, 151 (1998).

[17] K. Góral, K. Rzaźewski, and T. Pfau. Phys. Rev. A 61, 051601(R) (2000).

[18] S. Giovanazzi, A. Görlitz and T. Pfau. Phys. Rev. Lett. 89, 130401 (2002).

[19] B. C. Crooker, B. Hebral, E. N. Smith, Y. Takano, and J. D. Reppy. Phys. Rev. Lett. 51, 666 (1983).

[20] J. E. Lye, L. Fallani, M. Modugno, D. S. Wiersma, C. Fort, and M. Inguscio. Phys. Rev. Lett. 95, 070401 (2005).

[21] D. Clement, A. F. Varon, J. A. Retter, L. SanchezPalencia, A. Aspect, and P. Bouyer. New J. Phys. 8, 165 (2006).

[22] L. Sanchez-Palencia, D. Clement, P. Lugan, P. Bouyer, and A. Aspect. New J. Phys. 10, 045019 (2008).

[23] J. Billy, V. Josse, Z. Zuo, A. Bernard, B. Hambrecht, P. Lugan, D. Clement, L. Sanchez-Palencia, P. Bouyer, and A. Aspect. Nature 453, 891 (2008).

[24] P. Krüger, L. M. Andersson, S. Wildermuth, S. Hofferberth, E. Haller, S. Aigner, S. Groth, I. Bar-Joseph, and J. Schmiedmayer. Phys. Rev. A 76, 063621 (2007).

[25] J. Fórtagh and C. Zimmermann. Rev. Mod. Phys. 79, 235 (2007).

[26] U. Gavish and Y. Castin. Phys. Rev. Lett. 95, 020401 (2005).

[27] B. Gadway, D. Pertot, J. Reeves, M. Vogt, and D. Schneble. Phys. Rev. Lett. 107, 145306 (2011).

[28] B. Damski, J. Zakrzewski, L. Santos, P. Zoller, and M. Lewenstein. Phys. Rev. Lett. 91, 080403 (2003).

[29] T. Schulte, S. Drenkelforth, J. Kruse, W. Ertmer, J. Arlt, K. Sacha, J. Zakrzewski, and M. Lewenstein. Phys. Rev. Lett. 95, 170411 (2005).

[30] G. Roati, C. D'Errico, L. Fallani, M. Fattori, C. Fort, M. Zaccanti, G. Modugno, M. Modugno, and M. Inguscio. Nature 453, 895 (2008).

[31] K. Huang and H. F. Meng. Phys. Rev. Lett. 69, 644 (1992).

[32] S. Giorgini, L. Pitaevskii, and S. Stringari. Phys. Rev. B 49, 12938 (1994).
[33] A. V. Lopatin and V. M. Vinokur. Phys. Rev. Lett. 88, 235503 (2002).

[34] G. M. Falco, A. Pelster, and R. Graham. Phys. Rev. A 75, 063619 (2007).

[35] R.Graham and A. Pelster. Functional Integral Approach to Disordered Bosons. World Scientific (2007)

[36] M. Kobayashi and M. Tsubota. Phys. Rev. B 66, 174516 (2002).

[37] C. Krumnow and A. Pelster. Phys. Rev. A 84, 021608(R) (2011).

[38] B. Abdullaev and A. Pelster. Eur. Phys. J. D 66, 314 (2012).

[39] B. Nikolic, A. Balaz, and A. Pelster. Phys. Rev. A 88, 013624 (2013).

[40] K. Glaum, A. Pelster, H. Kleinert, and T. Pfau. Phys. Rev. Lett. 98, 080407 (2007).

[41] K. Glaum and A. Pelster. Phys. Rev. A 76, 023604 (2007).

[42] A. R. P. Lima and A. Pelster. Phys. Rev. A 84, 041604(R) (2011).

[43] A. R. P. Lima and A. Pelster. Phys. Rev. A 86, 063609 (2012).

[44] N. N. Bogoliubov. J. Phys.(USSR) 11, 4 (1947).

[45] N. M. Hugenholtz, and D. Pines. Phys. Rev. 116, 489 (1959).

[46] Y. Nambu. Phys. Rev. Lett. 4, 380 (1960).

[47] J. Goldstone. Nuovo Cim. 19, 154 (1961).

[48] T. D. Lee, K. Huang, and C. N. Yang. Phys. Rev. 106, 1135 (1957).

[49] C. Gaul and C. A. Müller. Phys. Rev. A 83, 063629 (2011).

[50] C. A. Müller and C. Gaul. New J. Phys. 14, 075025 (2012).

[51] J. O. Andersen. Rev. Mod. Phys. 76, 599 (2004).

[52] I. S. Gradshteyn and I. M. Ryzhik, Table of Integrals, Series, and Products, 7th ed. (Academic Press, New York, 2007).

[53] A. L. Fetter and J. D. Walecka, Quantum Theory of Many- Particle Systems, (Dover, New York, 2003).

[54] H. Kleinert and V. Schulte-Frohlinde, Critical Properties of $\phi^{4}$-Theories, (World Scientific, Singapore, 2001).

[55] E. Timmermans, P. Tommasini, and K. Huang, Phys. Rev. A 55, 3645 (1997).

[56] G. M. Falco, A. Pelster, and R. Graham, Phys. Rev. A 76, 013624 (2007).

[57] M. Ueda, Fundamentals and New Frontiers of BoseEinstein Condensation (World Scientific, Singapore, 2010).

[58] R. Graham and A. Pelster. Int. J. Bif. Chaos 19, 2745 (2009).

[59] E. Lifshitz and L. Pitaevskii, Statistical Physics, Part 2 (Elsevier, Oxford, 2003).

[60] C. A. Müller. arxiv: 1409.6987. 